\title{
Phase jump method for efficiency enhancement in free-electron lasers
}

\author{
Alan Mak, Francesca Curbis, and Sverker Werin \\ MAX IV Laboratory, Lund University, P.O. Box 118, SE-22100 Lund, Sweden \\ (Received 15 November 2016; published 22 June 2017; corrected 8 November 2017)
}

\begin{abstract}
The efficiency of a free-electron laser can be enhanced by the phase jump method. The method utilizes the phase-shifting chicanes in the drift sections between the undulator segments. By applying appropriate phase jumps, the microbunched electron beam can decelerate and radiate coherently beyond the initial saturation, enabling further energy transfer to the optical beam. This article presents a new physics model for the phase jump method, and supports it with numerical simulations. Based on the electron dynamics in the longitudinal phase space, the model describes the energy extraction mechanism, and addresses the selection criteria for the phase jump magnitude. While the ponderomotive bucket is stationary, energy can be extracted from electrons outside the bucket. With the aid of the new model, a comparison is made between the phase jump method and undulator tapering. The model also explores the potential of the phase jump method to suppress the growth of synchrotron sidebands in the optical spectrum.
\end{abstract}

DOI: 10.1103/PhysRevAccelBeams.20.060703

\section{INTRODUCTION}

In most single-pass free-electron laser (FEL) facilities, the undulator line is segmented by drift sections, where instruments for beam focusing, trajectory correction and diagnostics are installed. Often time, phase shifters are also installed in the drift sections [1-4].

Conventional phase shifters are compact magnetic chicanes, made up of either permanent magnets or electromagnets. The magnetic chicane can be used to increase the electron path length in the drift section, thus adjusting the phase angle between the electron beam and the optical wave.

A common application of phase shifters is phase correction, equalizing the phase angles at the two ends of a drift section. This allows the optical waves emitted in different undulator segments to interfere constructively. Without the phase correction, the velocity difference between electrons and light in the drift section can lead to an unwanted change in the phase angle, causing the optical waves to interfere destructively.

Another application of phase shifters is the suppression of the fundamental wavelength in harmonic lasing, thereby increasing the spectral intensity of the desired harmonic $[5,6]$. In addition, the phase shifter is an essential element of the mode-locking technique [7] for the generation of attosecond optical pulses.

Moreover, phase shifters can be used for further energy extraction beyond the initial saturation, and hence the

*alan.mak@maxiv.lu.se

Published by the American Physical Society under the terms of the Creative Commons Attribution 4.0 International license. Further distribution of this work must maintain attribution to the author(s) and the published article's title, journal citation, and DOI. enhancement of the FEL efficiency. This is achieved by choosing appropriate phase jumps, and purposely altering the phase angle between the electron beam and the optical wave. This phase jump method was first proposed by Varfolomeev et al. [8], and can be used in place of the technique of undulator tapering [9-11].

The phase jump method was subsequently studied by Ratner et al. [12]. The study exploits the mathematical equivalence of phase jumps and undulator tapering, and relies on a preoptimized taper to deduce the required phase jumps.

In this article, we further the study of the phase jump method by developing a physics model independent of undulator tapering. Our intuitive model illustrates the particle dynamics in the longitudinal phase space, and enables a deeper understanding of the energy extraction mechanism.

In Sec. II, we start with the steady-state model, focusing on a single ponderomotive bucket. We propose the microbunch deceleration cycle, and describe the energy extraction both in and out of the bucket.

In relation to this mechanism, we discuss the selection criteria for each phase jump, the requirement on the undulator segment length, and the origin of the final saturation. We then compare and contrast our phase jump model with the classic Kroll-Morton-Rosenbluth (KMR) model [9] of undulator tapering.

In Sec. III, we verify the features of our steady-state model by means of a steady-state numerical simulation.

In Sec. IV, we expand the steady-state model to include time-dependent effects. In particular, we discuss the potential of the phase jump method to suppress the growth of sidebands in the optical spectrum.

In Sec. V, we perform a time-dependent simulation to demonstrate that the phase jump method remains valid in 


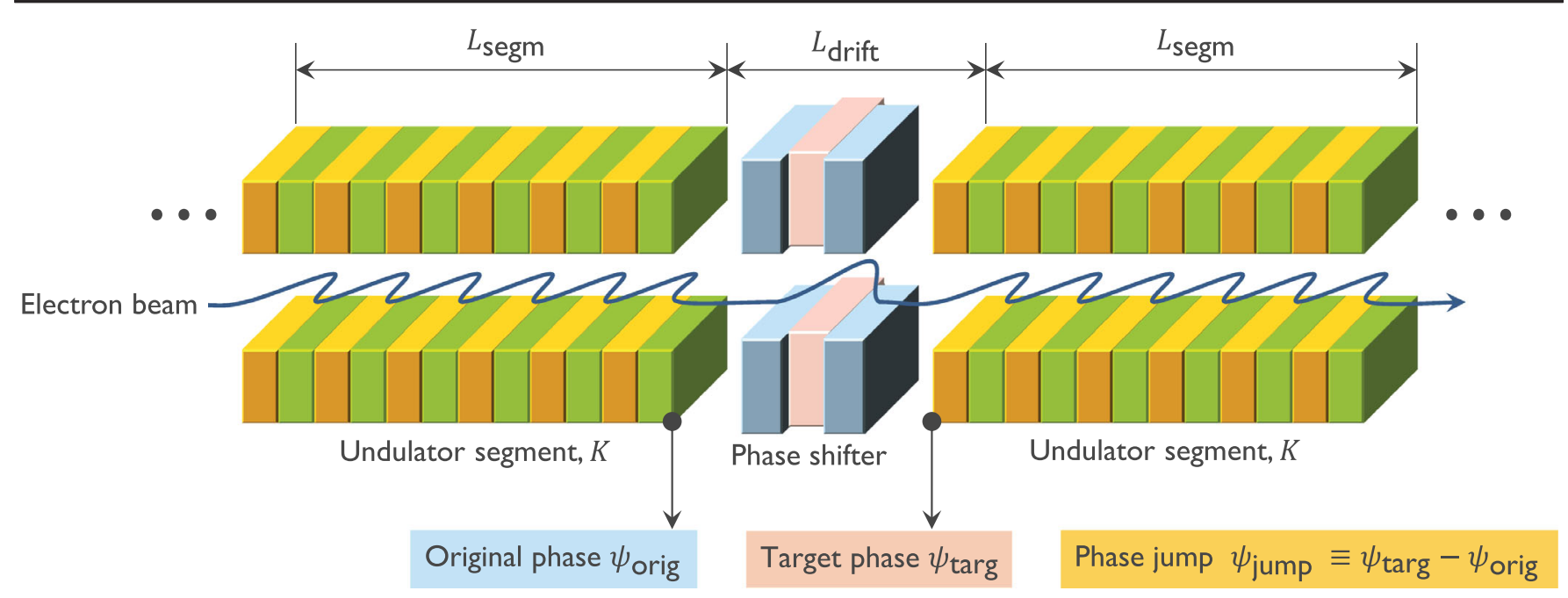

FIG. 1. Schematic diagram showing two of the undulator segments and the drift section in between. The phase shifter in the drift section can increase the electron path length, thus adjusting the phase angle between the electron beam and the optical wave. Focusing magnets, corrector magnets and diagnostics instruments are also commonly installed in drift sections, but are not shown in this diagram.

the presence of time-dependent effects, and to demonstrate that the phase jump method is more effective than undulator tapering in sideband suppression.

\section{STEADY-STATE MODEL}

\section{A. Problem description}

Consider an FEL with a planar undulator line segmented by drift sections, as depicted in Fig. 1. All undulator segments have the same length $L_{\text {segm }}$, and all drift sections have the same length $L_{\text {drift }}$. In every drift section, there is a phase shifter installed. The distance between the centers of every two adjacent phase shifters is $L_{\mathrm{segm}}+L_{\text {drift }}$, which is constant.

In this section, we develop a one-dimensional, steadystate model of the phase jump method, which makes use of the phase shifters to sustain the growth of radiation power at the fundamental wavelength beyond the initial saturation point.

In particular, we are interested in the effect of the phase jumps alone, in the absence of undulator tapering. We therefore restrict ourselves to a constant undulator period $\lambda_{u}=2 \pi / k_{u}$ and a constant undulator parameter

$$
K=\frac{e B_{0}}{m_{e} c k_{u}}
$$

where $e$ is the absolute value of the electron charge, $m_{e}$ is the electron rest mass, $c$ is the speed of light, and $B_{0}$ is the peak undulator field.
In addition, we assume the amplitude $E_{0}$ and phase $\phi$ of the optical field to be slowly varying in the course of the FEL interaction.

\section{B. Energy definitions}

The energy of an electron can be expressed as $\gamma m_{e} c^{2}$, and the resonant energy $\gamma_{R} m_{e} c^{2}$ is defined by

$$
\gamma_{R}=\sqrt{\frac{\lambda_{u}}{2 \lambda}\left(1+\frac{K^{2}}{2}\right)},
$$

where $\lambda=2 \pi / k$ is the radiation wavelength.

In the case of monochromatic seeding, $\lambda$ is the wavelength of the seed radiation, and $\gamma_{R}$ is determined by $\lambda$ through Eq. (2). In the case of self-amplified spontaneous emission (SASE), $\gamma_{R}$ is determined by the energy of the incoming electron beam, and $\lambda$ is determined by $\gamma_{R}$ through Eq. (2).

With undulator tapering, the undulator parameter $K$ decreases with increasing distance $z$ along the undulator line. According to the definition in Eq. (2), $\gamma_{R}$ decreases along $z$ to retain the radiation wavelength $\lambda$. Without undulator tapering, however, $K$ is constant. According to the definition in Eq. (2), $\gamma_{R}$ remains constant to retain $\lambda$.

In other words, the resonant energy is constant in the phase jump method. This allows us to express the energy of an electron as the relative deviation from the resonant energy, by the variable

$$
\eta \equiv \frac{\gamma-\gamma_{R}}{\gamma_{R}} .
$$


Even though the resonant energy is constant by definition, if the electrons themselves can decrease in $\eta$ beyond the initial saturation point, they can continue to transfer energy to the optical field.

\section{Phase definitions}

The longitudinal profile of the electron bunch is customarily treated in slices of width $\lambda$. During the FEL interaction, each slice contains a pondermotive potential well. In the steady-state model, all slices are assumed to be identical, and it suffices to consider only a single slice.

In the analysis of phase jumps, we are not interested in the absolute phase of an electron. Instead, it is more convenient to consider the phase of an electron relative the ponderomotive potential well, with the usual definition

$$
\psi \equiv\left(k+k_{u}\right) z-\omega t+\phi .
$$

Within a single slice, $\psi$ spans from $-\pi$ to $\pi$.

As indicated in Fig. 1, we let $\psi_{\text {orig }} \in[-\pi, \pi]$ be the original phase of an electron at the starting point of a drift section, and $\psi_{\operatorname{targ}} \in[-\pi, \pi]$ be the target phase at the end point of the same drift section. We then define the phase jump as the difference:

$$
\psi_{\text {jump }}=\psi_{\text {targ }}-\psi_{\text {orig }} \in[-2 \pi, 2 \pi] .
$$

Note that $\psi_{\text {jump }}=0$ does not mean that the phase shifter is turned off. Instead, it means that the phase shifter is configured merely for phase correction, whereby the phase angle between the electron and the optical field at the beginning of the drift section is preserved at the end of the drift section.

Note also the sign convention that a positive $\psi_{\text {jump }}$ corresponds to shifting the electron forward in $\psi$. However, a conventional phase shifter applies the phase jump by increasing the electron path length. It can only shift electrons backward in $\psi$, but not forward. In practice, if the required $\psi_{\text {jump }}$ is positive, we need to shift the electron backward to another potential well by a phase of $2 n \pi-\psi_{\text {jump }}$, where $n$ is a positive integer.

As a side note, this phase jump method for efficiency enhancement can, in principle, be implemented in combination with the iSASE technique [13] for bandwidth reduction. This is done by choosing a large $n$, so that the optical field emitted by the electrons toward the tail of the bunch may develop correlations with the electrons toward the head of the bunch, thus increasing the coherence length.

\section{Equations of motion}

In our model of the phase jump method, we concern ourselves with the electron dynamics in the longitudinal phase space $(\psi, \eta)$. In the undulator segments, the longitudinal dynamics of an electron can be described by two equations of motion:

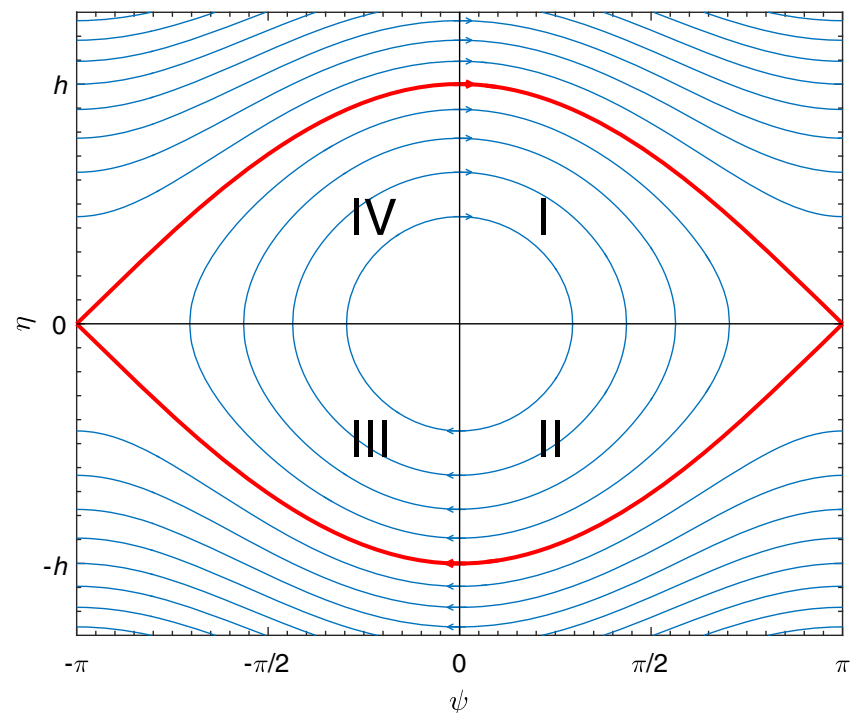

FIG. 2. The longitudinal phase space $(\psi, \eta)$, with electron trajectories shown by the blue curves. The red curve is the separatrix, and the region enclosed by it is the ponderomotive bucket. The straight lines $\eta=0$ and $\psi=0$ divide the space into four quadrants, as indicated by the Roman numerals.

$$
\begin{aligned}
& \frac{d \eta}{d z}=-\frac{\Omega^{2}}{2 k_{u}} \sin \psi, \\
& \frac{d \psi}{d z}=2 k_{u} \eta .
\end{aligned}
$$

A derivation of these equations is given in Ref. [14]. In Eq. (6), the quantity

$$
\Omega=\sqrt{\frac{e}{m_{e} c^{2}} \frac{k_{u} K f_{B} E_{0}}{\gamma_{R}^{2}}} \propto \sqrt{E_{0}}
$$

is the angular frequency of the synchrotron oscillation, which has the dimension of inverse length. Meanwhile, $f_{B}=J_{0}(\xi)-J_{1}(\xi)$ is the Bessel factor for planar undulators, with $\xi=K^{2} /\left[2\left(K^{2}+2\right)\right]$. By substituting Eq. (6) into the derivative of Eq. (7), we can verify that the longitudinal dynamics satisfies the pendulum equation

$$
\frac{d^{2} \psi}{d z^{2}}+\Omega^{2} \sin \psi=0
$$

\section{E. Phase space trajectories}

The equations of motion (6) and (7) satisfy the Hamilton equations for the Hamiltonian

$$
H(\psi, \eta)=c k_{u} \eta^{2}+\frac{c \Omega^{2}}{2 k_{u}}(1-\cos \psi) .
$$

The electron trajectories in the longitudinal phase space $(\psi, \eta)$ are given by the level set of the function $H(\psi, \eta)$, and are shown in Fig. 2. 
In particular, the trajectory highlighted in red is known as the separatrix. Along the separatrix, the Hamiltonian has the value

$$
H_{\text {sep }}=H( \pm \pi, 0)=\frac{c \Omega^{2}}{k_{u}} .
$$

The region enclosed by the separatrix is known as the ponderomotive bucket. Within the bucket, $H<H_{\text {sep}}$. The trajectories are closed orbits, and the electrons are trapped. Outside the bucket, $H>H_{\text {sep }}$. The trajectories are unbounded, and the electrons are untrapped.

The maximum $\eta$ value along the separatrix gives the half-height of the bucket,

$$
h=\frac{\Omega}{k_{u}} .
$$

Invoking the definition of the synchrotron frequency in Eq. (8), we obtain the proportionality

$$
h \propto \sqrt{E_{0}},
$$

meaning that the bucket half-height $h$ increases with the optical field amplitude $E_{0}$.

In Fig. 2, the horizontal line $\eta=0$ and the vertical line $\psi=0$ divide the longitudinal phase space into four quadrants, as indicated by the Roman numerals.

In quadrants I and II, $\psi>0$. In quadrants III and IV, $\psi<0$. According to Eq. (6), this implies $d \eta / d z<0$ in quadrants I and II, and $d \eta / d z>0$ in quadrants III and IV. In other words, electrons decelerate in quadrants I and II, and accelerate in quadrants III and IV. Due to the conservation of energy, energy is transferred to the optical field in quadrants I and II, and energy is absorbed from the optical field in quadrants III and IV.

In quadrants I and IV, $\eta>0$. In quadrants II and III, $\eta<0$. According to Eq. (7), this implies that electrons have increasing $\psi$ in quadrants I and IV, and decreasing $\psi$ in quadrants II and III.

\section{F. Phase jump commencement}

The essence of the phase jump method is microbunch deceleration. The aim is to decelerate the microbunched beam after the initial saturation, so that it can continue to radiate coherently. Thus, the phase jumps should commence in the vicinity of the initial saturation point, where the microbunching is fully developed.

In the exponential regime, the phase shifters should be configured for $\psi_{\text {jump }}=0$, or there will be disruption in the microbunch development. For SASE FELs in particular, applying $\psi_{\text {jump }} \neq 0$ in the exponential regime can also lead to a red or blue shift in the radiation wavelength, depending on the magnitudes of the phase jumps and their positions along the undulator line [6].

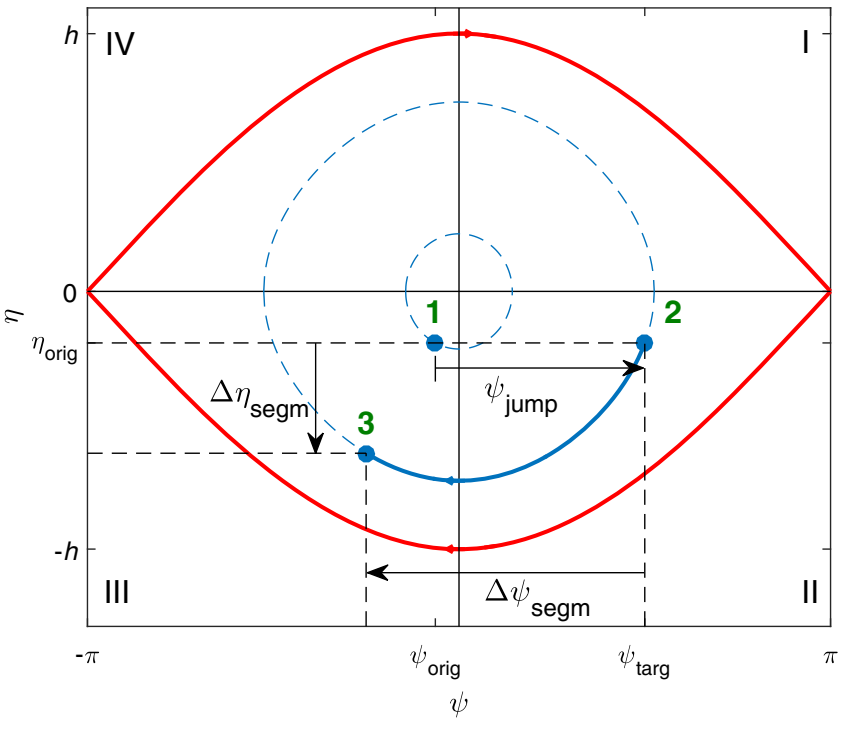

FIG. 3. The microbunch deceleration cycle as illustrated by the movement of the average particle within the ponderomotive bucket. Position 1 corresponds to the starting point of a drift section, position 2 the end point of the same drift section, and position 3 the end point of the subsequent undulator segment.

\section{G. Microbunch deceleration mechanism}

To analyze the microbunch deceleration, it is convenient to follow the motion of an average particle within the microbunch $\mu$. Let $(\bar{\psi}, \bar{\eta})$ be the coordinates of the average particle in the longitudinal phase space. They can be defined as

$$
\begin{aligned}
& \bar{\eta} \equiv\langle\eta\rangle_{\mu}=\frac{1}{N} \sum_{j \in \mu}^{N} \eta_{j}, \\
& \bar{\psi} \equiv \arg \left\langle e^{-i \psi}\right\rangle_{\mu}=-i \ln \left(\frac{1}{N} \sum_{j \in \mu}^{N} e^{-i \psi_{j}}\right),
\end{aligned}
$$

where $N$ is the number of particles in $\mu$.

Microbunch deceleration takes place in quadrants I and II, where particles decelerate and transfer energy to the optical field (see Fig. 2). Thus, a general principle of the phase jump method is to increase the time that the average particle spends in the deceleration quadrants (I and II), and decrease the time that the average particle spends in the acceleration quadrants (III and IV).

In the longitudinal phase space $(\psi, \eta)$, a phase jump moves a particle horizontally. If the average particle lies in quadrant IV, then we should choose a phase jump that moves it into quadrant I. If it lies in quadrant III, then we should move it into quadrant II. If it lies in quadrant II but is about to enter quadrant III, then we should move it to a slightly larger phase within quadrant II (i.e. away from quadrant III).

The mechanism is illustrated in Fig. 3. Suppose that the average particle has an original phase $\bar{\psi}=\psi_{\text {orig }}<0$ at the 
starting point of a drift section, as indicated by position 1 . We then apply a phase jump $\psi_{\text {jump }}>0$, so that the average particle arrives at a target phase $\bar{\psi}=\psi_{\text {targ }}>0$ at the end point of the drift section, as indicated by position 2 .

In an ideal phase shifter, electrons have no energy loss to synchrotron radiation, and the average particle has the same $\eta$ at position 2 as at position 1.

At position 2, the electrons enter an undulator segment, and follow the phase space trajectories described by Eqs. (6) and (7). In particular, the average particle follows the solid blue curve. So long as $\bar{\psi}>0$, the microbunch decelerates, and transfers energy to the optical field.

The average particle then arrives at position 3, as it reaches the end point of the undulator segment. Depending on the length $L_{\text {segm }}$ of the undulator segment, position 3 can be located in either quadrant II or III. In quadrant III, where $\bar{\psi}<0$, the microbunch absorbs energy from the optical field.

Within the undulator segment, $\bar{\psi}$ and $\bar{\eta}$ of the average particle have changed by $\Delta \psi_{\text {segm }}$ and $\Delta \eta_{\text {segm }}$, respectively. Provided that $\Delta \eta_{\text {segm }}<0$, the microbunch has a net deceleration, and hence a net energy transfer to the optical field.

In the course of the energy transfer, the optical field amplitude $E_{0}$ changes. Consequently, the bucket halfheight $h$ changes according to the proportionality (13). In principle, this can also lead to a distortion of the particle trajectories in the longitudinal phase space $(\psi, \eta)$. However, since $E_{0}$ is assumed to be slowly varying, the distortion of the solid blue curve is negligible as the average particle transits from position 2 to position 3 .

The end point of the undulator segment is also the start point of the next drift section. We can then repeat this microbunch deceleration cycle, by taking position 3 of the old cycle as position 1 of the new cycle. The cycle can continue until the end of the last undulator segment.

As the cycle continues, the microbunch moves towards the bottom of the ponderomotive bucket. Close to the bottom of the bucket, further phase jumps will move the microbunch out of the bucket. In other words, we can divide the energy extraction process beyond the initial saturation point into three main stages: (i) the in-bucket regime, (ii) the out-of-bucket regime, and (iii) the final saturation regime.

With an appropriate choice of the target phase $\psi_{\text {targ }}$ in every phase jump, we can have $\Delta \eta_{\text {segm }}<0$ in every undulator segment between the initial saturation and the final saturation. Obtaining the precise value of the optimal $\psi_{\text {targ }}$ is a matter of empirical phase scan. But from a theoretical perspective, there are general criteria for a good choice of $\psi_{\text {targ }}$ within the deceleration quadrants.

\section{H. In-bucket regime}

The in-bucket regime is the first stage beyond the initial saturation point. At this stage, microbunch deceleration takes place along the closed orbits within the ponderomotive bucket.

\section{Lower bound for good target phase}

In the single-cycle microbunch deceleration illustrated in Fig. 3, the energy extraction is the most efficient if the average particle stays within the deceleration quadrants throughout the entire undulator segment, and never manages to enter quadrant III. For this to be the case, the chosen target phase $\psi_{\text {targ }}$ must satisfy the criterion

$$
\begin{aligned}
\psi_{\mathrm{targ}}-\left|\Delta \psi_{\text {segm }}\right| & \geq 0 \\
\Leftrightarrow \psi_{\mathrm{targ}} & \geq\left|\Delta \psi_{\text {segm }}\right| .
\end{aligned}
$$

In order to proceed from here, we obtain an expression for $\Delta \psi_{\text {segm }}$ by integrating both sides of Eq. (7) with respect to $z$ over one undulator segment. This yields

$$
\left|\Delta \psi_{\mathrm{segm}}\right|=2 k_{u}\left|\int_{z^{\prime}}^{z^{\prime}+L_{\mathrm{segm}}} \bar{\eta}(z) d z\right|
$$

Within the undulator segment, we expect the average particle to decelerate, and $\bar{\eta}(z)$ should therefore be more negative than the original value $\eta_{\text {orig }}$ right before the undulator segment. Hence,

$$
\left|\Delta \psi_{\text {segm }}\right| \geq 2 k_{u}\left|\eta_{\text {orig }}\right| L_{\text {segm }} .
$$

Combining the inequalities (16) and (18), we obtain the lower bound $\psi_{\min }$ for the choice of $\psi_{\mathrm{targ}}$ :

$$
\psi_{\text {targ }} \geq 2 k_{u}\left|\eta_{\text {orig }}\right| L_{\text {segm }} \equiv \psi_{\text {min }} .
$$

When configuring each phase shifter, $\psi_{\text {targ }}$ needs to be at least $\psi_{\min }$, for the average particle to have a chance of avoiding the acceleration quadrants. If $\psi_{\mathrm{targ}}$ is less than $\psi_{\text {min }}$, then the average particle will definitely enter quadrant III within the upcoming undulator segment.

\section{Upper bound for good target phase}

During the in-bucket regime, we should keep the average particle in the bucket as long as possible. This allows us to fully exploit the in-bucket regime before the average particle becomes detrapped.

Thus, the upper bound $\psi_{\max }$ for the target phase $\psi_{\text {targ }}$ is given by the separatrix in the deceleration quadrants (see Fig. 3). This can be expressed mathematically as

$$
H\left(\psi_{\text {max }}, \eta_{\text {orig }}\right)=H_{\text {sep }} .
$$

To proceed from Eq. (20), we can substitute the righthand side by Eq. (11), and the left-hand side by Eq. (10) with $(\psi, \eta)=\left(\psi_{\text {max }}, \eta_{\text {orig }}\right)$. Cognizant of the fact that 
$0 \leq \psi_{\max } \leq \pi$, we can then solve for $\psi_{\max }$, and obtain the expression

$$
\psi_{\max }=2 \arccos \left(\frac{k_{u}^{2}\left|\eta_{\text {orig }}\right|}{\Omega^{2}}\right) .
$$

This is the upper bound for the choice of $\psi_{\mathrm{targ}}$, in order to avoid entering the out-of-bucket regime.

\section{Undulator segment length}

Within the first few cycles of the mechanism depicted in Fig. 3, the average particle should have reached the $\eta<0$ region, i.e., quadrants II and III. For the microbunch deceleration to be efficient, the average particle should stay within quadrant II, without entering quadrant III. However, this will not be possible if $\left|\Delta \psi_{\text {segm }}\right|$ is too large (see Fig. 3). From the inequality (18), we notice that $\left|\Delta \psi_{\text {segm }}\right|$ increases with the undulator segment length $L_{\text {segm. }}$. This imposes an upper limit on $L_{\text {segm }}$.

As a particle undergoes one complete orbit in the ponderomotive bucket, it travels down the undulator magnet by a distance of one synchrotron period $L_{\text {sync }}=2 \pi / \Omega$. As the particle sweeps across one quadrant in the bucket, it undergoes a quarter of a complete orbit, and travels down the undulator magnet by a distance of $L_{\text {sync }} / 4$.

Thus, for the average particle to stay within a single quadrant (namely, quadrant II), an undulator segment should be no longer than $L_{\text {sync }} / 4$. Since $L_{\text {sync }}$ varies with $z$, the requirement for the undulator segment length is

$$
L_{\text {segm }}<\frac{1}{4} \min \left[L_{\text {sync }}(z)\right] .
$$

\section{Regime transition}

As the microbunch deceleration cycle continues, the relative energy deviation $\bar{\eta}$ of the average particle becomes more and more negative, meaning that $\left|\eta_{\text {orig }}\right|$ becomes larger with every phase jump.

Throughout the in-bucket regime, $\psi_{\min }$ increases with $\left|\eta_{\text {orig }}\right|$ according to Eq. (19), and $\psi_{\max }$ decreases with increasing $\left|\eta_{\text {orig }}\right|$ according to Eq. (21). As the average particle is close to the bottom of the bucket, we will eventually encounter a scenario where $\psi_{\min }>\psi_{\max }$.

In such a scenario, it is no longer possible to choose a target phase $\psi_{\text {targ }}$ in the range $\psi_{\text {min }} \leq \psi_{\text {targ }} \leq \psi_{\max }$. We are then forced to choose $\psi_{\operatorname{targ}}>\psi_{\max }$, and move the average particle out of the bucket. This marks the end of the in-bucket regime, and the beginning of the out-of-bucket regime.

\section{J. Out-of-bucket regime}

In the out-of-bucket regime, the trajectories in the longitudinal phase space $(\psi, \eta)$ are unbounded. Nonetheless, microbunch deceleration is possible. The mechanism is

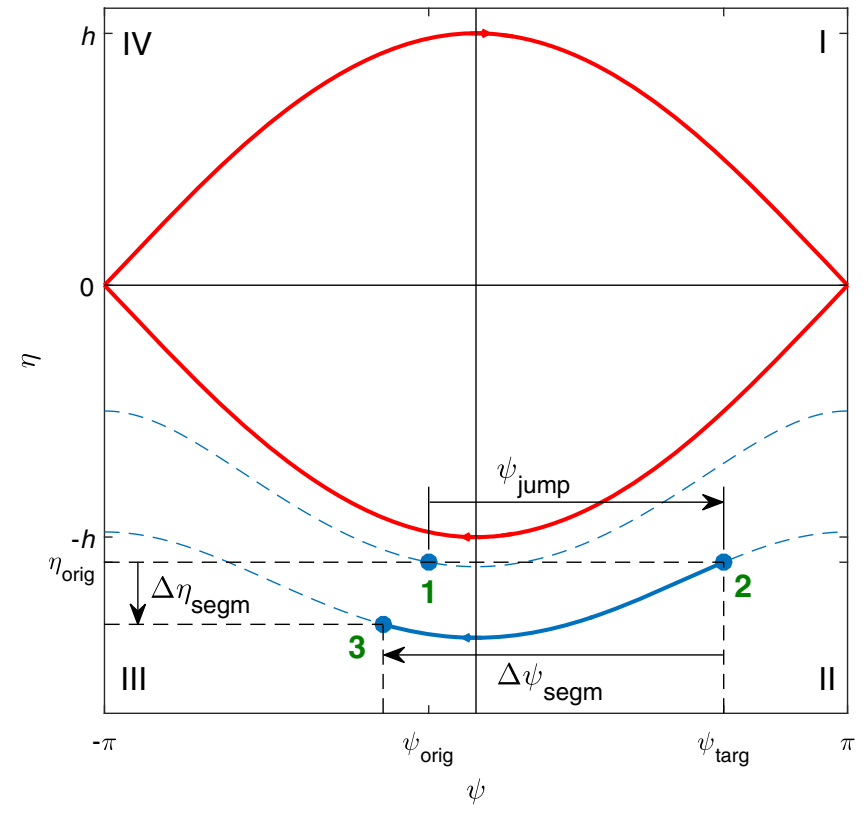

FIG. 4. The microbunch deceleration cycle as illustrated by the movement of the average particle outside the bucket. Position 1 corresponds to the starting point of a drift section, position 2 the end point of the same drift section, and position 3 the end point of the subsequent undulator segment.

similar to that in the in-bucket regime, and is illustrated in Fig. 4.

\section{Deceleration efficiency}

The deceleration efficiency in each undulator segment depends on the slope of the particle trajectory in the deceleration quadrants of the $(\psi, \eta)$ space. The steeper is the slope, the higher is the rate at which a particle loses energy.

The slope is given by the derivative $d \eta / d \psi$. Dividing Eq. (6) by Eq. (7), we can obtain an expression for the derivative as follows:

$$
\left|\frac{d \eta}{d \psi}\right|=\frac{\Omega^{2}}{4 k_{u}^{2}|\eta|} \sin \psi .
$$

Note that in the deceleration quadrants, we have $0 \leq \psi \leq \pi$ and hence $0 \leq \sin \psi \leq 1$. As a result, $|d \eta / d \psi|$ is inversely proportional to $|\eta|$, and the deceleration efficiency decreases with increasing $|\eta|$.

For the in-bucket regime, $|\eta|<h$. For the out-of-bucket regime, $|\eta|>h$. Thus, the deceleration efficiency is lower in the out-of-bucket regime than in the in-bucket regime. As the microbunch deceleration cycle continues, the deceleration efficiency decreases with every undulator segment.

\section{Lower bound for good target phase}

For the in-bucket regime, the deceleration in an undulator segment can be made more efficient by keeping the 
average particle in the deceleration quadrants and preventing it from entering the acceleration quadrants. This argument also applies to the out-of-bucket regime. Thus, the lower bound for a good $\psi_{\operatorname{targ}}$ in the out-of-bucket regime is also given by Eq. (19):

$$
\psi_{\text {min }}=2 k_{u}\left|\eta_{\text {orig }}\right| L_{\text {segm }} .
$$

\section{Upper bound for good target phase}

In the out-of-bucket regime, the average particle is already outside the bucket. The separatrix does not impose any limit on $\psi_{\text {targ. }}$. In principle, the upper limit of $\psi_{\text {targ }}$ in the out-of-bucket regime is $\pi$, which is the maximum phase in the deceleration quadrants.

However, it is not favorable to let the average particle get too close to $\pi$, or a fraction of the particles within the microbunch will leak into the $\pi<\psi<3 \pi$ region, which corresponds to the acceleration quadrants associated with the bucket ahead. In that region, particles absorb energy from the optical field.

The precise upper bound for the choice of $\psi_{\text {targ }}$ depends on the $\psi$ spread of the microbunch. But roughly speaking, the upper bound for a good $\psi_{\operatorname{targ}}$ is slightly below $\pi$.

\section{K. Final saturation regime}

According to the relation (18), $\left|\Delta \psi_{\text {segm }}\right|$ increases with $\left|\eta_{\text {orig }}\right|$. At some point in the out-of-bucket regime, $\left|\eta_{\text {orig }}\right|$ will have become so large that

$$
\left|\Delta \psi_{\text {segm }}\right|=\pi
$$

This signifies the onset of the final saturation regime.

Beyond that point, it is no longer possible to prevent the average particle from moving into quadrant III within a single undulator segment, regardless of the choice of $\psi_{\mathrm{targ}}$ (see Fig. 4). The microbunch deceleration cycle then becomes inefficient, and $\Delta \eta_{\text {segm }}$ approaches zero.

As $\Delta \eta_{\text {segm }}$ approaches zero, the inequality (18) can be approximated by

$$
\left|\Delta \psi_{\text {segm }}\right| \approx 2 k_{u}\left|\eta_{\text {orig }}\right| L_{\text {segm }} \equiv \psi_{\text {min }}
$$

With this approximation, the relative energy deviation at the onset of the final saturation regime is then

$$
\left|\eta_{\text {orig }}\right|=\frac{\pi}{2 k_{u} L_{\text {segm }}} .
$$

The final saturation point is reached when $\Delta \eta_{\text {segm }} \geq 0$, i.e. when it is no longer possible to maintain a net transfer of energy from microbunch to the optical field.

\section{Small subtlety about phase jump}

When using a nonisochronous phase shifter, it is important to note that the specified phase jump is only valid for particles at a certain reference energy. In other words, a particle which is not at the reference energy experiences a different phase jump from the specified value.

In applying the phase jump method, it is convenient to use the resonant energy $\gamma_{R} m_{e} c^{2}$ as the reference energy, as it is constant. But in our model, we are mainly concerned about the phase jump applied to the average particle, which has $\gamma \neq \gamma_{R}$ in general. Therefore, we need a conversion formula between the phase jump $\psi_{\text {jump }}^{\mathrm{A}}$ for the average particle and the phase jump $\psi_{\text {jump }}^{\mathrm{R}}$ for particles at the resonant energy.

To obtain such a conversion formula, we model the phase-shifting chicane as a one-period undulator, with undulator period $\hat{\lambda}_{u}$ and deflection parameter $\hat{K}$. After the one undulator period, the slippage $\hat{\lambda}$ is given by

$$
\hat{\lambda}=\frac{\hat{\lambda}_{u}}{2 \gamma^{2}}\left(1+\frac{\hat{K}^{2}}{2}\right)
$$

For particles at the resonant energy, $\gamma=\gamma_{R}$. The slippage $\hat{\lambda}$ is related to the phase jump $\psi_{\text {jump }}^{\mathrm{R}}$ by

$$
\frac{\psi_{\mathrm{jump}}^{\mathrm{R}}}{2 \pi}=-\frac{\hat{\lambda}}{\lambda},
$$

where $\lambda$ (without the caret) is the actual radiation wavelength of the FEL. The negative sign in the equation arises from the sign convention of $\psi_{\text {jump }}^{\mathrm{R}}$.

In general, the average particle has $\gamma \neq \gamma_{R}$. In the same one-period undulator, the average particle experiences a different slippage $\hat{\lambda}+\Delta \hat{\lambda}$, which is related to the phase jump $\psi_{\text {jump }}^{\mathrm{A}}$ by

$$
\frac{\psi_{\text {jump }}^{\mathrm{A}}}{2 \pi}=-\frac{\hat{\lambda}+\Delta \hat{\lambda}}{\lambda} .
$$

To proceed from here, we take the differential on both sides of Eq. (27), and obtain

$$
\Delta \hat{\lambda}=\frac{\hat{\lambda_{u}}}{2}\left(1+\frac{\hat{K}^{2}}{2}\right)\left(\frac{-2 \Delta \gamma}{\gamma^{3}}\right)=-2 \hat{\lambda} \frac{\Delta \gamma}{\gamma}=-2 \hat{\lambda} \bar{\eta} .
$$

As usual, $\bar{\eta}$ is the relative energy deviation of the average particle. Using Eqs. (28), (29), and (30), we can eliminate $\lambda$, $\hat{\lambda}$, and $\Delta \hat{\lambda}$. This results in the conversion formula

$$
\psi_{\text {jump }}^{\mathrm{A}}=\psi_{\text {jump }}^{\mathrm{R}}(1+2 \bar{\eta}) .
$$

\section{Comparison with undulator tapering}

Apart from the phase jump method, undulator tapering is another common technique for efficiency enhancement in FELs. In this subsection, we compare and contrast the two techniques. In particular, we discuss the similarities and differences between our phase jump model and the classic 
Kroll-Morton-Rosenbluth (KMR) model [9] of undulator tapering.

Both models make use of one-dimensional Hamiltonian mechanics to describe the particle dynamics in the longitudinal phase space $(\psi, \eta)$. The KMR tapering model follows the motion of a resonant particle, which defines the stable point in the middle of the ponderomotive bucket. Our phase jump model follows the motion of the average particle within the microbunch.

In the KMR model, we directly control the undulator parameter $K$, and move the resonant particle vertically in the $(\psi, \eta)$ space. In our phase jump model, we directly control the phase jump $\psi_{\text {jump }}$, and move the average particle horizontally in the $(\psi, \eta)$ space.

In both models, the energy extraction is sustained beyond the initial saturation point by bringing a fraction of particles toward lower energies. As these particles decelerate, energy is transferred to the optical field, due to the conservation of energy.

However, the underlying principle of the particle deceleration is different in the two models. In the KMR model, particle deceleration relies on the deceleration of the bucket itself. In our phase jump model, particle deceleration relies on the microbunch deceleration cycle.

In the KMR model, the bucket moves toward lower energies during the energy extraction. In this process, the phase of the resonant particle increases, and the width of the bucket decreases. In our phase jump model, the bucket is stationary, and does not reduce in width. During the energy extraction process, the optical field amplitude increases, and the height of the bucket increases.

In the KMR model, particles need to be trapped in the bucket in order to decelerate. In our phase jump model, the microbunch deceleration cycle continues in the out-ofbucket regime. Energy extraction outside the bucket is impossible for the former, but possible for the latter.

In the KMR model, the efficiency of particle deceleration is determined by the rate at which the bucket decreases in energy. This, in turn, depends on the $d K / d z$, the rate at which the undulator parameter decreases along the undulator line. In our phase jump model, the efficiency of particle deceleration is determined by $d \eta / d \psi$, the slope of the particle trajectory in the longitudinal phase space. This, in turn, depends on the relative energy deviation $\bar{\eta}$ of the average particle, as evidenced by Eq. (23).

In the KMR model, the undulator segment length required for the efficient deceleration of particles is $L_{\text {segm }}<$ $\min \left[L_{\text {sync }}(z)\right]$, as discussed in Ref. [11]. In our phase jump model, the requirement is $L_{\text {segm }}<\min \left[L_{\text {sync }}(z)\right] / 4$, which is a more stringent one.

In the KMR model, the main causes of the final saturation are the weakening of refractive guiding and the detrapping of particles. In our phase jump model, the main causes of the final saturation are the decrease of $|d \eta / d \psi|$ and increase of $\left|\Delta \psi_{\text {segm }}\right|$ with particle energy.
TABLE I. Parameters of the steady-state simulation.

\begin{tabular}{lcc}
\hline \hline Parameter & Symbol & Value \\
\hline Electron beam energy & $\gamma m_{e} c^{2}$ & $5 \mathrm{GeV}$ \\
Energy spread & $\sigma_{\gamma} / \gamma$ & $1 \times 10^{-4}$ \\
Beam current & $I$ & $3 \mathrm{kA}$ \\
Normalized emittance & $\varepsilon_{x, y}$ & $0.4 \mathrm{~mm} \mathrm{mrad}$ \\
Average of beta function & $\bar{\beta}_{x, y}$ & $7 \mathrm{~m}$ \\
Radiation wavelength & $\lambda$ & $2 \AA$ \\
Undulator period & $\lambda_{u}$ & $20 \mathrm{~mm}$ \\
Undulator parameter & $K$ & 1.35 \\
Length of each undulator segment & $L_{\text {segm }}$ & $1 \mathrm{~m}$ \\
Length of each drift section & $L_{\text {drift }}$ & $0.2 \mathrm{~m}$ \\
\hline \hline
\end{tabular}

\section{STEADY-STATE SIMULATION}

\section{A. Case definition}

So far, our physics model of the phase jump method is a one-dimensional and steady-state one. For the purpose of verifying the model, we perform a three-dimensional and steady-state simulation study, using the numerical simulation code GENESIS [15].

We first define a case for the simulation study. The main parameters are listed in Table I.

In the chosen case, SASE is the start-up mechanism of the FEL. The effective shot-noise power is $830 \mathrm{~W}$.

Planar undulator segments are used. Undulator tapering is not implemented.

The lattice for strong focusing is in a FODO configuration, with one quadrupole magnet in every other drift section. The length of the FODO cell is $4.8 \mathrm{~m}$. Within the cell, the centers of the two quadrupole magnets are separated by a distance of $2.4 \mathrm{~m}$. The length of each quadrupole magnet is $80 \mathrm{~mm}$.

The strengths of the quadrupole magnets and the initial twiss parameters are matched self-consistently to give the average beta $\bar{\beta}_{x, y}$ specified in Table I.

In the GENESIS simulation, we place a phase shifter in every drift section, by putting an $\mathrm{AD}$ element in the external magnet file. We control the phase jump by setting the $A D$ element to an appropriate value.

Here the undulator segment length of $L_{\text {segm }}=1 \mathrm{~m}$ is smaller than what is used in most existing FEL facilities. The small $L_{\text {segm }}$ is chosen to fulfill the requirement (22).

\section{B. Initial saturation}

In order to obtain information about the initial saturation point, we first run the simulation in the absence of phase jumps, by setting $\psi_{\text {jump }}=0$ for all drift sections.

The simulation shows that the initial saturation occurs at $z=38.2 \mathrm{~m}$ within the 32nd undulator segment. The saturation power is $2 \mathrm{GW}$. At the initial saturation point, the bunching factor $b=\left|\left\langle e^{-i \psi}\right\rangle\right|$ is the highest, and has a value of 0.4 . 


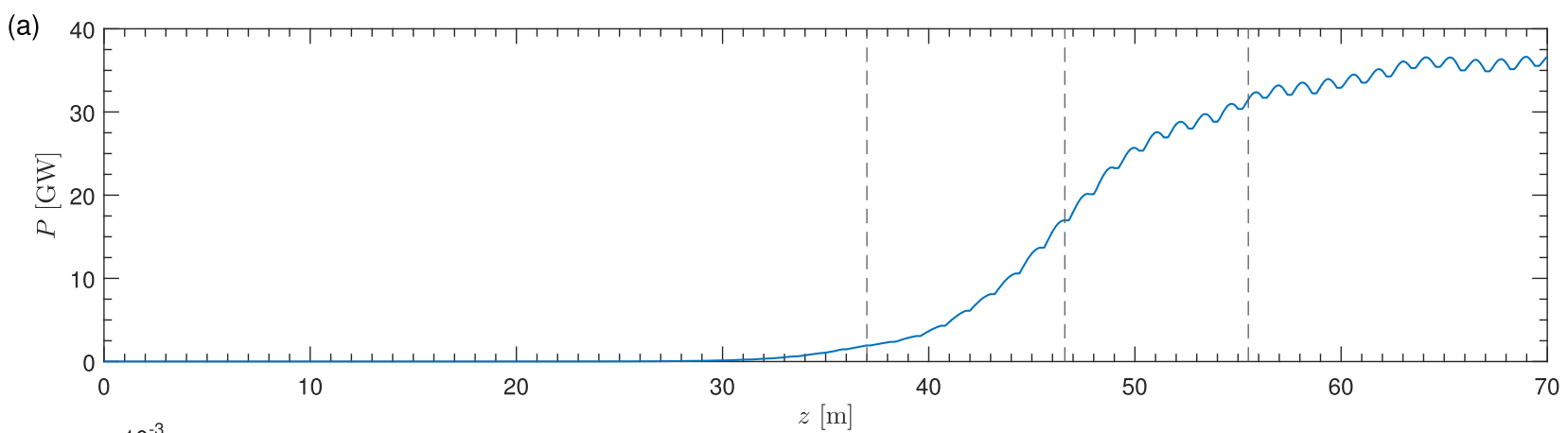

(b)
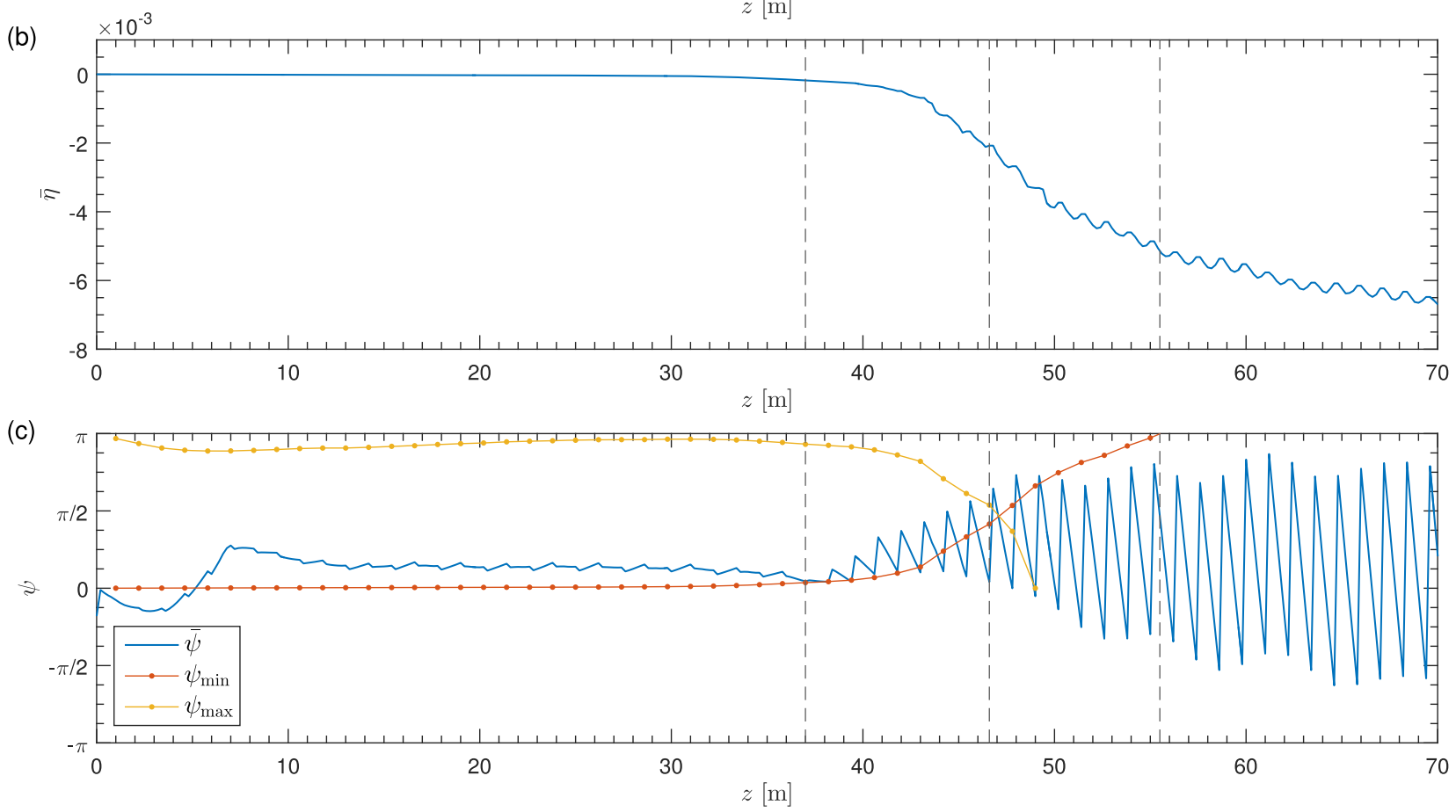

FIG. 5. Result of steady-state simulation. The following quantities are plotted as functions of the distance $z$ along the undulator line: (a) the radiation power, (b) the relative energy deviation $\bar{\eta}$ of the average particle, and (c) the ponderomotive phase $\bar{\psi}$ of the average particle (in blue), together with the lower bound $\psi_{\min }$ (in red) and the upper bound $\psi_{\text {max }}$ (in yellow) for the target phase $\psi_{\text {targ }}$ in each drift section. The dashed vertical lines mark the beginning of the in-bucket, out-of-bucket, and final saturation regimes.

\section{Phase jump commencement}

We then repeat the simulation with the introduction of phase jumps. We start the phase jumps in the vicinity of the initial saturation point, where the microbunching is fully developed. Thus, the first nonzero phase jump occurs in the drift section at $z=37 \mathrm{~m}$, immediately preceding the $32 \mathrm{nd}$ undulator segment. Meanwhile, we keep $\psi_{\text {jump }}=0$ for all the drift sections before $z=37 \mathrm{~m}$.

While there are infinite possible sets of phase jumps, we shall discuss one chosen set which yields an increase in radiation power beyond the initial saturation. The phase jumps are obtained by coarse tuning within the selection criteria discussed in Sec. II.

It is possible to obtain even higher radiation power by fine-tuning the phase jump. However, the purpose of this simulation study is to verify the steady-state model, and not to perform a thorough optimization.

\section{Radiation power evolution}

Figure 5(a) shows the radiation power $P$ as a function of $z$, for the chosen set of phase jumps. Along the radiation power curve, there are short, straight, horizontal sections, where the power is neither increasing nor decreasing. These sections correspond to the drift sections, where the radiation does not exchange energy with the electron beam.

After the first nonzero phase jump at $z=37 \mathrm{~m}$, the radiation power continues to grow. Up until $z=50 \mathrm{~m}$, the power grows monotonically. But after that, the power fluctuates. Within every undulator segment, the power first increases, and then decreases. Nonetheless, there is still a net power increase.

In the undulator segment which begins at $z=64.8 \mathrm{~m}$, there is no longer a net power increase within an undulator segment. This indicates the arrival of the final saturation. 
The power at the final saturation is $36 \mathrm{GW}$, which is 18 times the power at the initial saturation.

The FEL efficiency can be defined as the power ratio of the output radiation to the injected electron beam. With this definition, the chosen set of phase jumps enhance the FEL efficiency by a factor of 18 . It is possible to obtain an even larger enhancement factor by fine-tuning the phase jump values. But again, the purpose of this simulation study is to verify the model, and not to perform a thorough optimization.

\section{E. Energy evolution of the average particle}

We now turn our attention to the average particle within the microbunch. The relative energy deviation $\bar{\eta}$ of the average particle is defined by Eq. (14). Figure 5(b) shows $\bar{\eta}$ as a function of $z$.

Before the first nonzero phase jump at $z=37 \mathrm{~m}, \bar{\eta}$ remains close to zero, meaning that the energy of the average particle is close to the resonant energy.

After the first nonzero phase jump at $z=37 \mathrm{~m}, \bar{\eta}$ exhibits an overall decreasing trend. The energy of the average particle deviates further and further from the resonant energy. This is an evidence of microbunch deceleration.

As $\bar{\eta}$ becomes more and more negative, the rate of $\bar{\eta}$ decrease becomes lower and lower. This agrees with the prediction of Eq. (23).

Up until $z=50 \mathrm{~m}, \bar{\eta}$ decreases monotonically. But after that, $\bar{\eta}$ fluctuates. Comparing Fig. 5(a) and (b), we notice that a decrease in $\bar{\eta}$ corresponds to an increase in $P$, and vice versa. This can be explained by the conservation of energy. When $\bar{\eta}$ decreases, the microbunch loses energy. This energy is transferred to the radiation, leading to an increase in $P$.

The onset of the final saturation regime is defined by Eq. (26). According to this definition, the final saturation regime begins when $\bar{\eta}=5 \times 10^{-3}$. As seen in Fig. 5(b), this corresponds to $z=55.5 \mathrm{~m}$.

\section{F. Phase evolution of the average particle}

The ponderomotive phase $\bar{\psi}$ of the average particle is defined by Eq. (14). Figure 5(c) shows $\bar{\psi}$ as a function of $z$. After the first nonzero phase jump at $z=37 \mathrm{~m}, \bar{\psi}$ oscillates in $z$. The upward slopes coincide with the drift sections, while the downward slopes coincide with the undulator segments. In other words, a crest coincides with the start point of an undulator segment, while a trough coincides with the end point of an undulator segment.

In terms of the microbunch deceleration cycle (see Figs. 3 and 4), a crest corresponds to position 2, while a trough corresponds to position 1 or 3 . The period of the oscillation is one microbunch deceleration cycle. The crest value of each cycle is the target phase $\psi_{\text {targ }}$. The phase change represented by an upward slope is $\psi_{\text {jump }}$, while the phase change represented by a downward slope is $\Delta \psi_{\text {segm }}$.
Figure 5(c) also shows the lower bound $\psi_{\min }$ and the upper bound $\psi_{\max }$ for the target phase $\psi_{\text {targ }}$ in each drift section. The values are given by Eqs. (19) and (21). Recall that $\psi_{\max }$ is defined by the separatrix of the ponderomotive bucket in the $\psi \geq 0$ region, and that $\psi_{\min }$ is the minimum requirement for the average particle to avoid entering the $\psi<0$ region.

In Fig. 5(c), the region immediately after the first nonzero phase jump at $z=37 \mathrm{~m}$ is the in-bucket regime, as evidenced by the fact that $\bar{\psi}<\psi_{\max }$. The decrease of $\psi_{\max }$ along $z$ reflects that the average particle is moving towards the bottom of the bucket.

In the in-bucket regime, $\psi_{\text {targ }}$ is made to increase with $\psi_{\min }$, so as to fulfill the requirement that $\psi_{\operatorname{targ}}>\psi_{\min }$. As a result, the average particle is prevented from entering the $\psi<0$ region. Within every undulator segment, the average particle transfers energy to the radiation, without absorbing energy from the radiation. This explains the monotonic decrease of $\bar{\eta}$ [see Fig. 5(b)].

Prior to $z=46.5 \mathrm{~m}$, the choice of $\psi_{\text {targ }}$ satisfies the requirement $\psi_{\min }<\psi_{\text {targ }}<\psi_{\text {max }}$. But in the vicinity of $z=46.5 \mathrm{~m}$, the average particle is so close to the bottom of the bucket that we encounter the situation where $\psi_{\min } \approx \psi_{\max }$. We are then forced to choose $\psi_{\text {targ }}>\psi_{\max }$, thus placing the average particle outside the bucket. This marks the beginning of the out-of-bucket regime.

Even though the average particle is outside the bucket, a fraction of the particles in the microbunch are still inside the bucket. For the next two periods of the oscillations, a part of the microbunch follows the in-bucket trajectories, while a part of the microbunch follows the out-of-bucket trajectories. The average particle, tracing the average behavior of the entire microbunch, moves in and out of the bucket. At $z=49 \mathrm{~m}, \psi_{\max }=0$, indicating that the average particle is at the same energy level as the lowest point of the bucket.

In the out-of-bucket regime, $\psi_{\min }$ continues to increase, and becomes closer and closer to $\pi$. However, we want to prevent the average particle from getting too close to $\pi$, or a fraction of the particles in the microbunch will enter the acceleration region associated with the bucket ahead. This concern forces us to choose $\psi_{\mathrm{targ}}<\psi_{\min }$. The consequence is that the average particle enters the $\psi<0$ region, thus absorbing energy from the radiation. Hence, $\bar{\eta}$ no longer decreases monotonically [see Fig. 5(b)]. Instead, $\bar{\eta}$ decreases and increases within a single undulator segment.

The final saturation regime begins at $z=55.5 \mathrm{~m}$, where $\psi_{\min }=\pi$ [see Fig. 5(c)]. In this regime, it is no longer possible to prevent the average particle from entering the $\psi<0$ region, regardless of the choice of $\psi_{\text {targ. }}$. As the average particle enters deep into the $\psi<0$ region, the energy extraction becomes far less effective.

\section{G. Direct observation in phase space}

Next, we observe the microbunch deceleration cycle directly in the longitudinal phase space $(\psi, \eta)$. Figure 6 

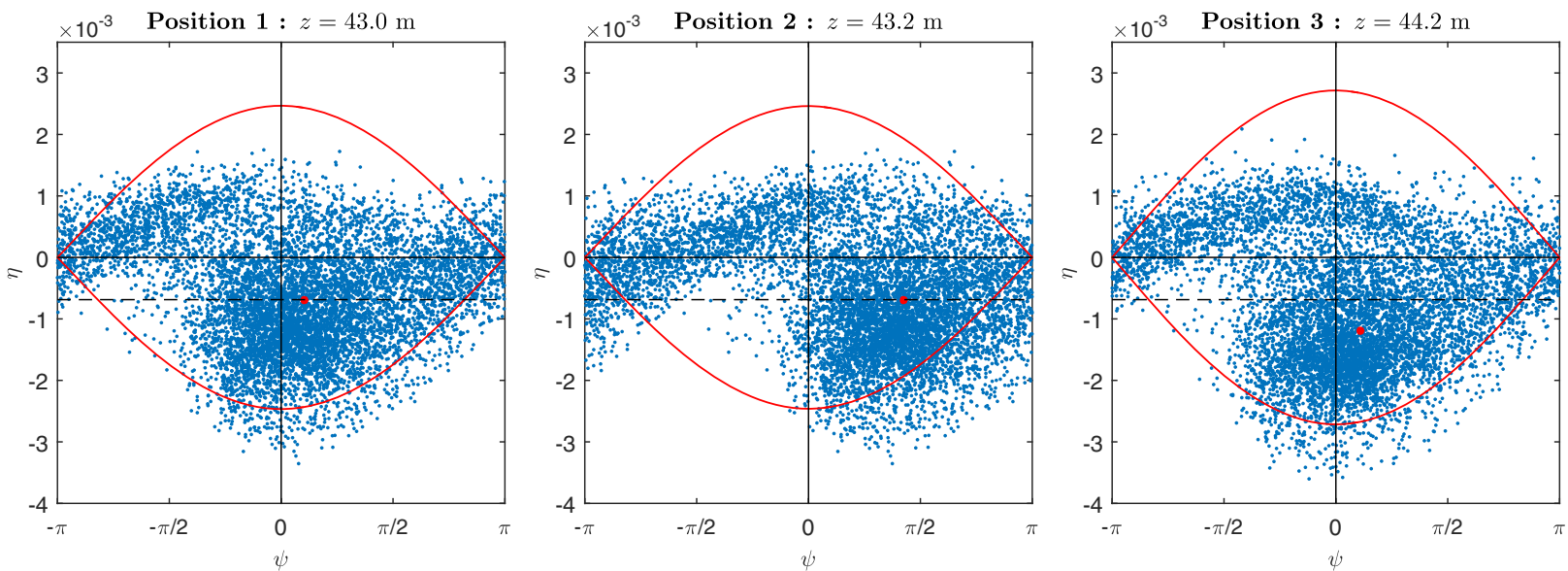

FIG. 6. Result of steady-state simulation. Snapshots in the longitudinal phase space $(\psi, \eta)$ showing a microbunch deceleration cycle in the in-bucket regime. The red dot represents the average particle within the microbunch.
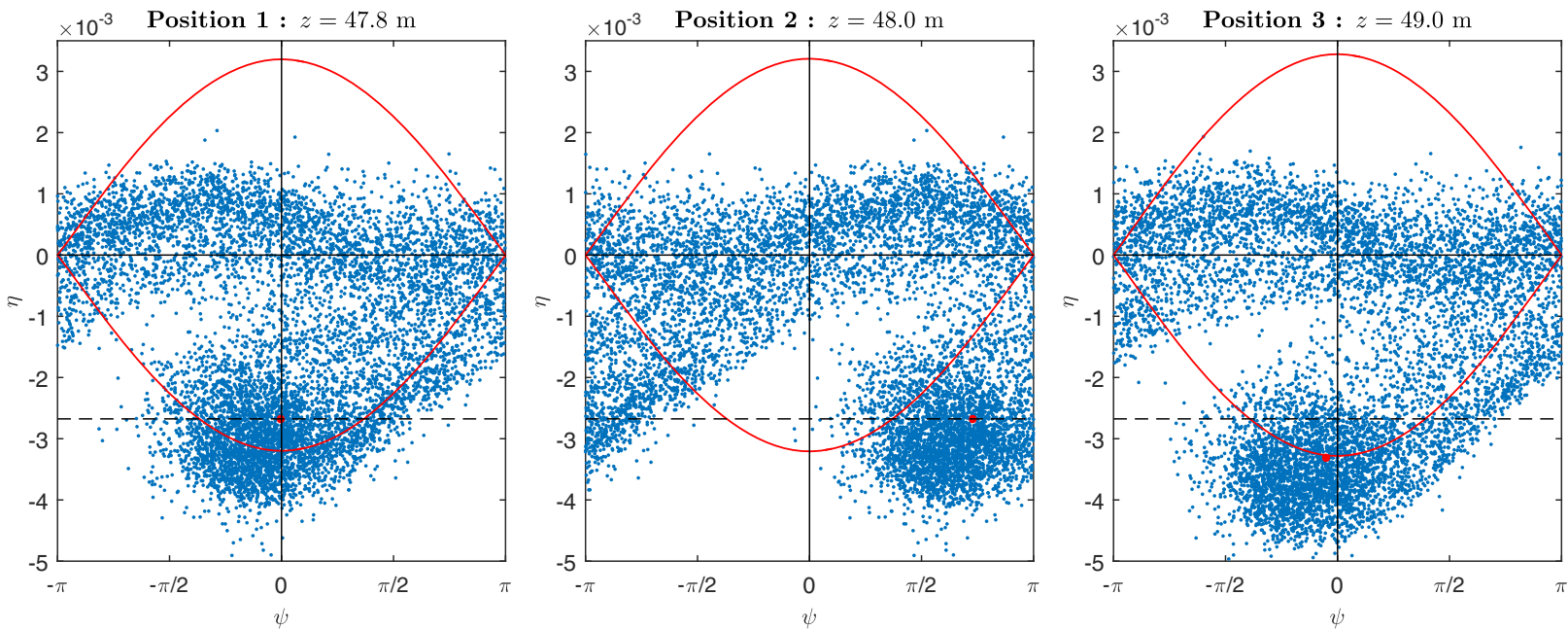

FIG. 7. Result of steady-state simulation. Snapshots in the longitudinal phase space $(\psi, \eta)$ showing a microbunch deceleration cycle during the transition from the in-bucket regime to the out-of-bucket regime.
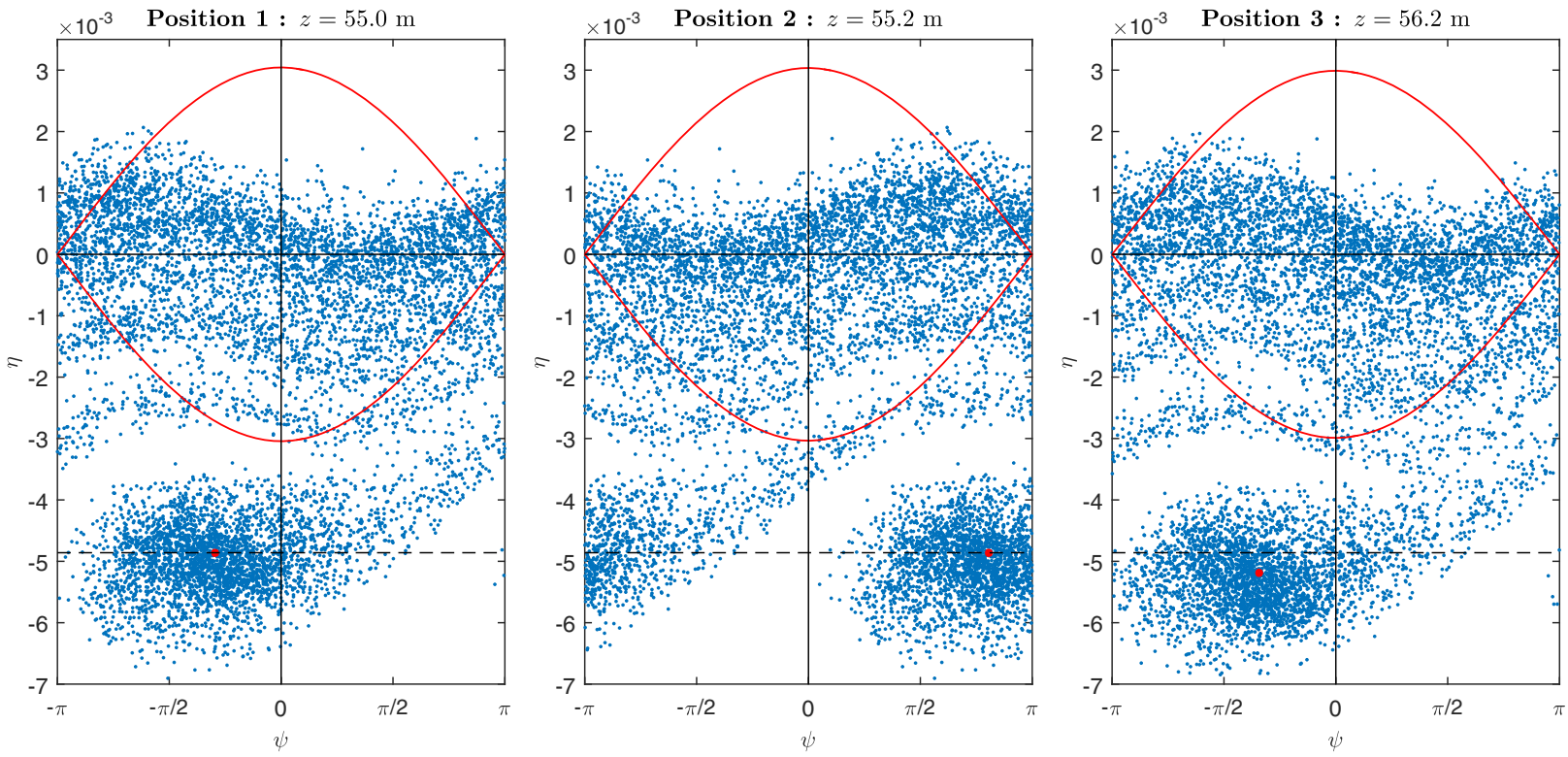

FIG. 8. Result of steady-state simulation. Snapshots in the longitudinal phase space $(\psi, \eta)$ showing a microbunch deceleration cycle at the onset of the final saturation regime. 
shows a cycle in the in-bucket regime. Figure 7 shows a cycle during the transition from the in-bucket regime to the out-of-bucket regime. Figure 8 shows a cycle at the onset of the final saturation regime.

In each of these phase space snapshots, the red curve represents the separatrix of the ponderomotive bucket, and the red dot represents the average particle. These snapshots clearly show that the electron beam remains microbunched along the undulator line. From these snapshots, it is also apparent that the microbunch moves toward lower $\eta$ as $z$ increases, verifying the microbunch deceleration once again.

In each cycle, position 1 corresponds to the starting point of the drift section, position 2 the end point of the drift section, and position 3 the end point of the subsequent undulator segment.

In position 1 and position 2 , the average particle has the same $\eta$. This is expected for an ideal phase shifter, wherein the phase jump $\psi_{\text {jump }}$ changes only the phase, but not the energy, of the average particle.

In the transition from position 2 to position 3 , the particles pass through an undulator segment, where there is energy exchange between the particles and the radiation. The energy exchange alters the bucket half-height $h$ slightly. This is also expected, as $h$ depends on the slowly varying optical field amplitude $E_{0}$ according to the proportionality (13).

In all the three cycles shown here, the average particle ends up with a lower $\eta$ at position 3 than at position 1, meaning that there is a net energy transfer from the microbunch to the radiation in the undulator segment. The motion of the average particle in these snapshots reflects the mechanism depicted in Figs. 3 and 4.

\section{H. Trace of the average particle}

Figure 9 shows the trace of the average particle in the longitudinal phase space $(\psi, \eta)$ over the entire undulator line. Within the trace, there are straight, horizontal sections, each representing the transition from position 1 to position 2 within a microbunch deceleration cycle.

As the average particle moves from high $\eta$ to lower $\eta$, the $\eta$ spacing between successive horizontal sections decreases, meaning that the amount of energy lost by the average particle decreases with every cycle. In other words, the deceleration efficiency decreases as $\eta$ becomes more and more negative, as predicted by Eq. (23).

At $\eta \approx-6 \times 10^{-3}$, the $\eta$ spacing between successive horizontal sections approaches zero. There is no longer a net energy transfer from the microbunch to the radiation. This indicates the arrival of the final saturation.

\section{Undulator segment length}

In the simulation, the synchrotron period $L_{\mathrm{sync}}$ varies as a function of $z$ (data not shown). The smallest value is $\min \left[L_{\mathrm{sync}}(z)\right]=5.9 \mathrm{~m}$, which occurs at $z=49 \mathrm{~m}$. With an

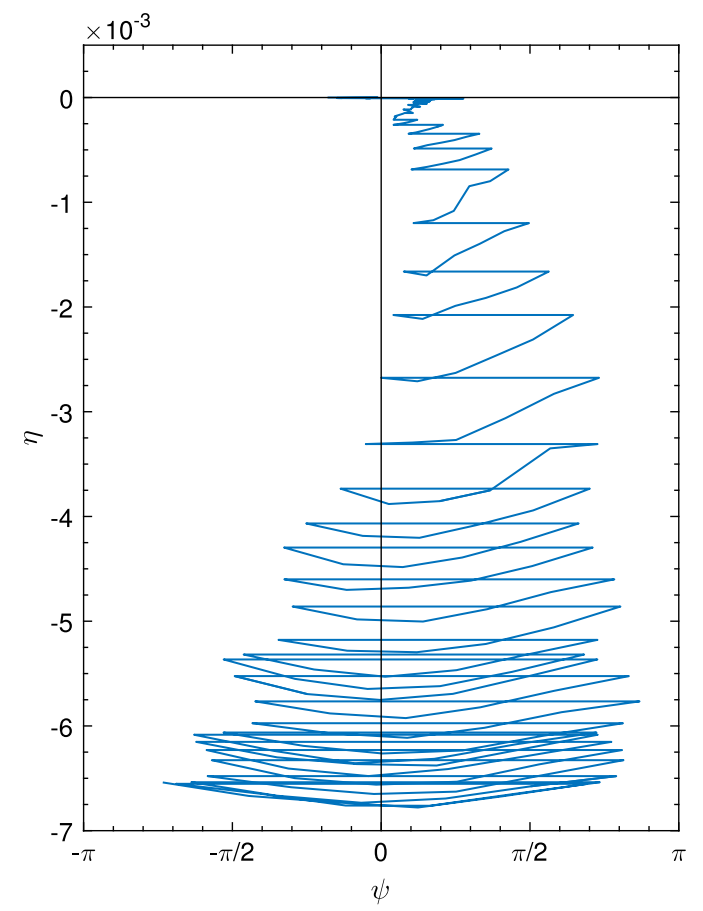

FIG. 9. Result of steady-state simulation. The trace of the average particle in the longitudinal phase space $(\psi, \eta)$ over the entire undulator line.

undulator segment length of $L_{\text {segm }}=1 \mathrm{~m}$ as specified in Table I, the requirement (22) is satisfied.

\section{TIME-DEPENDENT MODEL}

\section{A. Problem description}

In this section, we extend the steady-state model of the phase jump method into a time-dependent model. As before, we consider the longitudinal profile of the electron bunch as a series of slices, each with a width of $\lambda$.

The steady-state model assumes all slices to be identical. For every slice, the optical field that slips into the slice ahead is identical to the optical field that comes in from the slice behind. This implies that the electron bunch has an infinite length and a constant current. The resulting FEL signal has only a single frequency component, and hence zero bandwidth.

The time-dependent model departs from these assumptions, and accounts for the interactions between distinct slices in a profile of finite length. For example, in a Gaussian profile, the current $I$ at any position $t$ within the electron bunch is given by

$$
I(t)=I_{0} \exp \left(-\frac{t^{2}}{2 \sigma_{t}^{2}}\right)
$$

where $I_{0}$ is the peak current and $\sigma_{t}$ is the RMS bunch length. 


\section{B. Average average particle}

There is one average particle for every slice. In the steady-state model, all average particles behave in the exact same manner, as all slices are identical. The optimal phase jump for any one average particle is also the optimal for the entire electron bunch.

In the time-dependent model, however, all these average particles generally have distinct $(\bar{\psi}, \bar{\eta})$ values at any given position $z$ along the undulator line. The optimal phase jump for one average particle is generally not the optimal for another. In order to optimize the phase jump for the electron bunch as a whole, we need a compromise between all the average particles.

In obtaining the compromise, the average particles should not be treated equally, as the slices generally do not have the same charge. Thus, we propose to optimize the phase jump for an average average particle, which is the weighted average of all the average particles.

Let $(\overline{\bar{\psi}}, \overline{\bar{\eta}})$ be the coordinates of the average average particle in the longitudinal phase space. They can be defined as

$$
\begin{aligned}
& \overline{\bar{\eta}} \equiv \sum_{\mu} w_{\mu} \bar{\eta}_{\mu}, \\
& \overline{\bar{\psi}} \equiv \arg \left(\sum_{\mu} w_{\mu} e^{-i \bar{\psi}_{\mu}}\right),
\end{aligned}
$$

where

$$
w_{\mu}=\frac{I\left(t_{\mu}\right)}{\sum_{\nu} I\left(t_{\nu}\right)}
$$

is the weighting factor based on the slice current $I\left(t_{\mu}\right)$.

Note that the definitions (33), (34), and (35) are applicable to any electron profile, and not specifically to a Gaussian profile. With these definitions, the higher is the current in a slice, the better is the slice represented by the average average particle.

\section{Ansatz for optimization}

In the steady-state model, the average particle of a single slice is put through the microbunch deceleration cycles depicted in Figs. 3 and 4. The deceleration of the average particle is optimized by choosing an appropriate target phase $\bar{\psi}=\psi_{\text {targ }}$ for each cycle.

In the time-dependent model, this same mechanism is applicable to the average average particle of the entire electron bunch. The deceleration of the average average particle is optimized by choosing an appropriate target phase $\overline{\bar{\psi}}=\psi_{\text {targ }}$ for each cycle.

For the average average particle, the in-bucket and outof-bucket regimes can be defined in relation to an imaginary bucket with half-height $h$ determined by the optical field amplitude $E_{0}$ averaged over all slices. The selection criteria for $\psi_{\text {targ }}$ then remain the same as in the steady-state model. In this subsection, we develop an ansatz for the optimal evolution of $\psi_{\text {targ }}$ along the undulator line, based on these selection criteria.

Among the selection criteria, the most important one is the common lower bound $\psi_{\min }$ in both the in-bucket and out-of-bucket regimes, given by Eq. (19). This criterion is a necessary condition for the microbunch to have a net deceleration, $\Delta \eta_{\text {segm }}<0$, within an undulator segment.

In contrast, the upper bound $\psi_{\max }$ in the in-bucket regime, given by Eq. (21), is not as crucial. Even if $\psi_{\text {targ }}$ exceeds $\psi_{\max }$ by a small amount, the energy extraction can still continue in the out-of-bucket regime.

According to Eq. (19), $\psi_{\min }$ increases with $|\overline{\bar{\eta}}|$ of the average average particle. Beyond the initial saturation point, $|\overline{\bar{\eta}}|$ increases gradually from zero as the electrons decelerate. Hence, $\psi_{\min }$ is expected to increase gradually from zero, and this is precisely the observation in the steady-state simulation [see Fig. 5(c)]. It is therefore logical that the optimal $\psi_{\text {targ }}$ should increase with every phase shift.

However, $\psi_{\text {targ }}$ cannot increase indefinitely, due to the upper bound in the out-of-bucket regime. The upper bound is a chosen value $\psi_{1}$, which is slightly less than $\pi$. This criterion is a necessary condition for preventing a microbunch from leaking into the acceleration quadrants associated with the bucket ahead. Therefore, the optimal $\psi_{\text {targ }}$ should increase gradually beyond the initial saturation point only until it reaches $\psi_{1}$, and remains at $\psi_{1}$ for all subsequent phase shifts.

We label the phase shifters along the undulator line with the index $m \in\{1,2, \ldots\}$. Suppose that the initial saturation point is in the vicinity of $m=m_{0}$. Then, all the phase shifters with $1 \leq m \leq m_{0}$ should be configured for $\psi_{\text {jump }}=0$, or equivalently, $\psi_{\text {targ }}=\psi_{\text {orig }}$. For $m>m_{0}$, we propose the following ansatz for the optimal evolution of $\psi_{\text {targ }}$ :

$\psi_{\operatorname{targ}}(m)= \begin{cases}\psi_{1} \times \frac{m-m_{0}}{m_{1}-m_{0}} & \text { for } m_{0}<m<m_{1} \\ \psi_{1} & \text { for } m \geq m_{1} .\end{cases}$

The growth of $\psi_{\text {targ }}$ is chosen to be linear for simplicity, and the phase shifter $m=m_{1}$ defines where $\psi_{\text {targ }}$ reaches $\psi_{1}$ for the first time.

The expression (36) can be regarded as a functional form, with every value of the parameter $m_{1}$ yielding a different function $\psi_{\operatorname{targ}}(m)$.

\section{Sideband suppression}

Sideband instability is a known issue of the FEL. It was first predicted theoretically [16] and observed experimentally [17] in the 1980s. 
The origin of sideband instability is the synchrotron oscillations during the FEL interaction. In the longitudinal phase space $(\psi, \eta)$, trapped electrons orbit around the stable centroid of the bucket, thus oscillating back and forth in $\psi$ at an angular frequency $\Omega$ given by Eq. (8). These oscillations give rise to a longitudinal modulation of amplitude and phase in the optical pulse.

The modulation manifests itself in the FEL spectrum as sidebands at parasitic wavelengths. The wavelength offset of these sidebands from the desired central wavelength $\lambda$ is given by [16]

$$
\frac{\Delta \lambda}{\lambda}= \pm \frac{\lambda_{u}}{L_{\mathrm{sync}}}
$$

where $L_{\text {sync }}=2 \pi / \Omega$ is the synchrotron period.

When the sidebands are amplified, a significant fraction of optical power is drawn from the central wavelength to the parasitic wavelengths. This degrades the spectral purity and the efficiency of the FEL.

In the steady-state model, the FEL efficiency is determined solely by the fraction of the electron beam's power that is converted into optical power. In the time-dependent model, the FEL efficiency is determined also by the power ratio between the central wavelength and the sidebands. Even with a high optical power extracted from the electron beam, the FEL can still be inefficient if much of the optical power is channeled to the sidebands instead of the central wavelength.

For undulator tapering, sideband growth can be significantly detrimental to the amplification of the central wavelength, especially when the post-saturation undulator section is multiple synchrotron periods long. This is discussed in Refs. [9,18,19].

While synchrotron oscillations are responsible for the sideband growth, the phase jump method has the potential to suppress the sideband growth by disrupting the synchrotron oscillations.

In the longitudinal phase space, a complete orbit within the bucket corresponds to a full period of synchrotron oscillation. In the in-bucket microbunch deceleration cycle depicted in Fig. 3, the trajectory of the particle from position 2 to position 3 is a portion of a complete orbit. In every cycle, the orbit is disrupted by the applied phase jump, which brings the particle from position 1 to position 2.

Provided that the undulator segment length $L_{\text {segm }}$ satisfies the requirement (22), the particle will never manage to make a complete orbit after the initial saturation.

\section{TIME-DEPENDENT SIMULATION}

\section{A. Case definition}

In this section, we conduct a three-dimensional and timedependent simulation study, using the same simulation
TABLE II. Parameters of the time-dependent simulation.

\begin{tabular}{lcc}
\hline \hline Parameter & Symbol & Value \\
\hline Electron beam energy & $\gamma m_{e} c^{2}$ & $16 \mathrm{GeV}$ \\
Energy spread & $\sigma_{\gamma} / \gamma$ & $1 \times 10^{-4}$ \\
Peak current & $I_{0}$ & $4 \mathrm{kA}$ \\
RMS bunch length & $\sigma_{t}$ & $1 \mu \mathrm{m}$ \\
Normalized emittance & $\varepsilon_{x, y}$ & $0.3 \mathrm{~mm} \mathrm{mrad}$ \\
Average of beta function & $\bar{\beta}_{x, y}$ & $10 \mathrm{~m}$ \\
Radiation wavelength & $\lambda$ & $1 \AA$ \\
Seed radiation power & $P_{\text {seed }}$ & $5 \mathrm{MW}$ \\
Undulator period & $\lambda_{u}$ & $40 \mathrm{~mm}$ \\
Default undulator parameter & $K$ & 2.79 \\
Length of each undulator segment & $L_{\text {segm }}$ & $4 \mathrm{~m}$ \\
Length of each drift section & $L_{\text {drift }}$ & $1 \mathrm{~m}$ \\
\hline \hline
\end{tabular}

code GENESIS [15]. In particular, we apply the ansatz (36) and optimize the phase jumps for the average average particle.

The purpose of this simulation study is twofold: (i) to demonstrate that the phase jump method remains valid when time-dependent effects are taken into account; and (ii) to compare the phase jump method to undulator tapering in terms of efficiency.

The case chosen for the simulation study is a seeded FEL, using longer undulator segments than in the previous simulation. The longitudinal profile of the electron bunch is a Gaussian function. The main parameters are listed in Table II.

To examine the performance of the FEL over a longer distance, we simulate the undulator line from $z=0$ up to $z=200 \mathrm{~m}$.

At $\lambda=1 \AA$, no suitable external laser is available. The seed radiation essentially needs to come from self-seeding. The production mechanism of the seed radiation is beyond the scope of this simulation study. Therefore, we input the seed radiation at $z=0$ without any regard to how it is produced.

The lattice for strong focusing is in a FODO configuration, with one quadrupole magnet in every drift section. The length of the FODO cell is $10 \mathrm{~m}$. Within the cell, the centers of the two quadrupole magnets are separated by a distance of $5 \mathrm{~m}$. The length of each quadrupole magnet is $80 \mathrm{~mm}$.

The strengths of the quadrupole magnets and the initial twiss parameters are matched self-consistently to give the average beta $\bar{\beta}_{x, y}$ specified in Table II.

\section{B. Optimization results}

The initial saturation point is located at $z=44 \mathrm{~m}$, i.e., the end of the 9th undulator segment, where the optical pulse energy is $22 \mu \mathrm{J}$.

For the phase jump method, we set $m_{0}=8$ in the ansatz (36), so that the first nonzero phase jump takes place 
immediately after the 9th undulator segment. Furthermore, we arbitrarily set $\psi_{1}=170^{\circ}=2.97 \mathrm{rad}$ for simplicity. We then scan the parameter $m_{1}$ for the highest optical pulse energy at the final saturation, and this optimization process yields $m_{1}=16$. The synchrotron period has a minimum value of $24 \mathrm{~m}$. With the undulator segment length of $4 \mathrm{~m}$, the requirement (22) is well satisfied.

For undulator tapering, we use the same starting point as for the phase jump method, so as to obtain a fair comparison. Immediately after the 9th undulator segment, the undulator parameter decreases quadratically with every segment, but remains uniform within every segment. We then scan the taper strength $\Delta K / K$ for the highest optical pulse energy at the final saturation, and this optimization process yields $\Delta K / K=8 \%$. The taper strength $\Delta K / K$ is defined such that $K$ is the default undulator parameter in resonance with the initial energy of the electron beam, and $K-\Delta K$ is the parameter of the last segment before $z=200 \mathrm{~m}$.

As a side note, if a SASE case were chosen instead of the seeded case, then the optical pulse energy would fluctuate from shot to shot. Regardless of the method of optimization, there is simply no way to find one set of phase jumps (or one undulator taper) that is optimal for every shot. The optimization for a SASE case would therefore require characterizing the statistical properties. This subject is beyond the scope of this article, and is left for future studies.

\section{Evolution of the optical pulse energy}

Figure 10 shows the evolution of the optical pulse energy with $z$ for the optimal phase jumps and the optimal taper. The dashed vertical line indicates the initial saturation point, which is the common starting point for the phase jump method and the undulator taper.

For the phase jump method, the final saturation occurs at $z=100 \mathrm{~m}$. The optical pulse energy at the final saturation is $0.53 \mathrm{~mJ}$, which is 24 times higher than that at the initial saturation. This enhancement in the optical pulse energy shows that the phase jump method remains valid in the presence of time-dependent effects.

For the undulator taper, the final saturation occurs at $z=130 \mathrm{~m}$. The optical pulse energy at the final saturation is $1.5 \mathrm{~mJ}$, which is three times the value for the phase jump method.

Comparing the undulator taper and the phase jump method, the former extracts a larger fraction of the electron beam's energy in the chosen case. However, this is true only when the undulator line is long enough for the energy extraction to reach the final saturation.

Immediately after the initial saturation, the phase jump method exhibits a faster growth in optical pulse energy (see Fig. 10). This is because the phase jump method allows particles to decelerate more rapidly in the region immediately following the initial saturation.

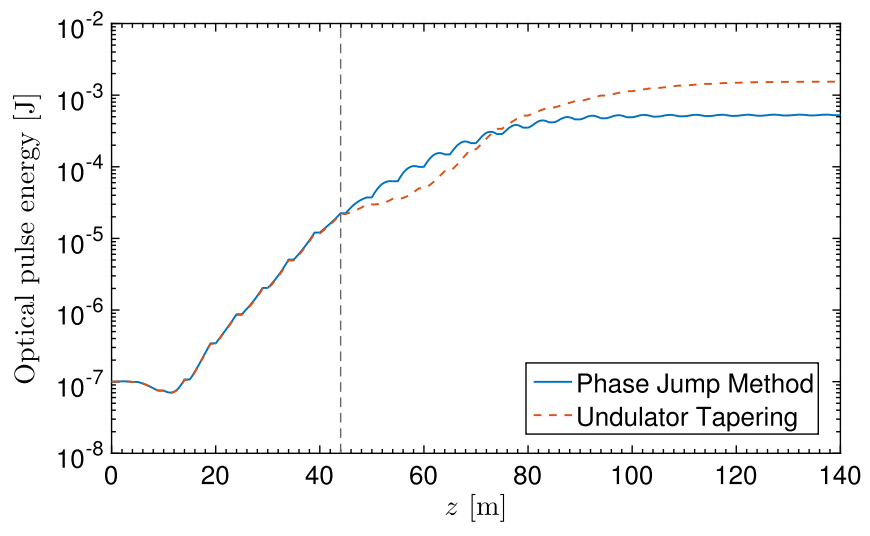

FIG. 10. Result of time-dependent simulation. The optical pulse energy is plotted as a function of the distance $z$ along the undulator line for the optimal phase jumps (blue) and the optimal taper (red). The dashed vertical line indicates the common starting point for the phase jump method and the taper. The vertical axis is on a logarithmic scale.

For tapering, the particle trapping region [10] comes right after the initial saturation point. In this region, a mild taper is needed to capture particles into the shrinking and descending bucket. A milder taper corresponds to a slower deceleration of the trapped particles.

In contrast, the phase jump method does not require such trapping process, as the microbunch deceleration cycle can take place both in and out of the bucket. Particles can be made to decelerate rapidly right after the initial saturation.

\section{Evolution of the optical spectrum}

Figure 11 illustrates the evolution of the optical spectrum for the optimal taper, with the snapshots at six positions along the undulator line. Figure 12 shows the corresponding snapshots for the optimal phase jumps.

In each snapshot, the spectral power is normalized to that at the central wavelength, to help compare the power ratio between the central wavelength and any sideband. Furthermore, the same binning for $\Delta \lambda / \lambda$ is used in all the snapshots.

In Fig. 11 for the optimal taper, snapshot (d) corresponds to the vicinity of the final saturation. Before the normalization, the absolute power at the central wavelength is $0.12 \mathrm{TW} / \mathrm{fm}$.

In Fig. 12 for the optimal phase jumps, snapshot (c) corresponds to the vicinity of the final saturation. Before the normalization, the absolute power at the central wavelength is $0.14 \mathrm{TW} / \mathrm{fm}$, close to the corresponding value for the optimal taper.

In the FEL interaction, it is the central wavelength that we intend to amplify. At the final saturation, even though the taper extracts more energy from the electron beam, it does not channel more energy to the central wavelength than the phase jumps do. 

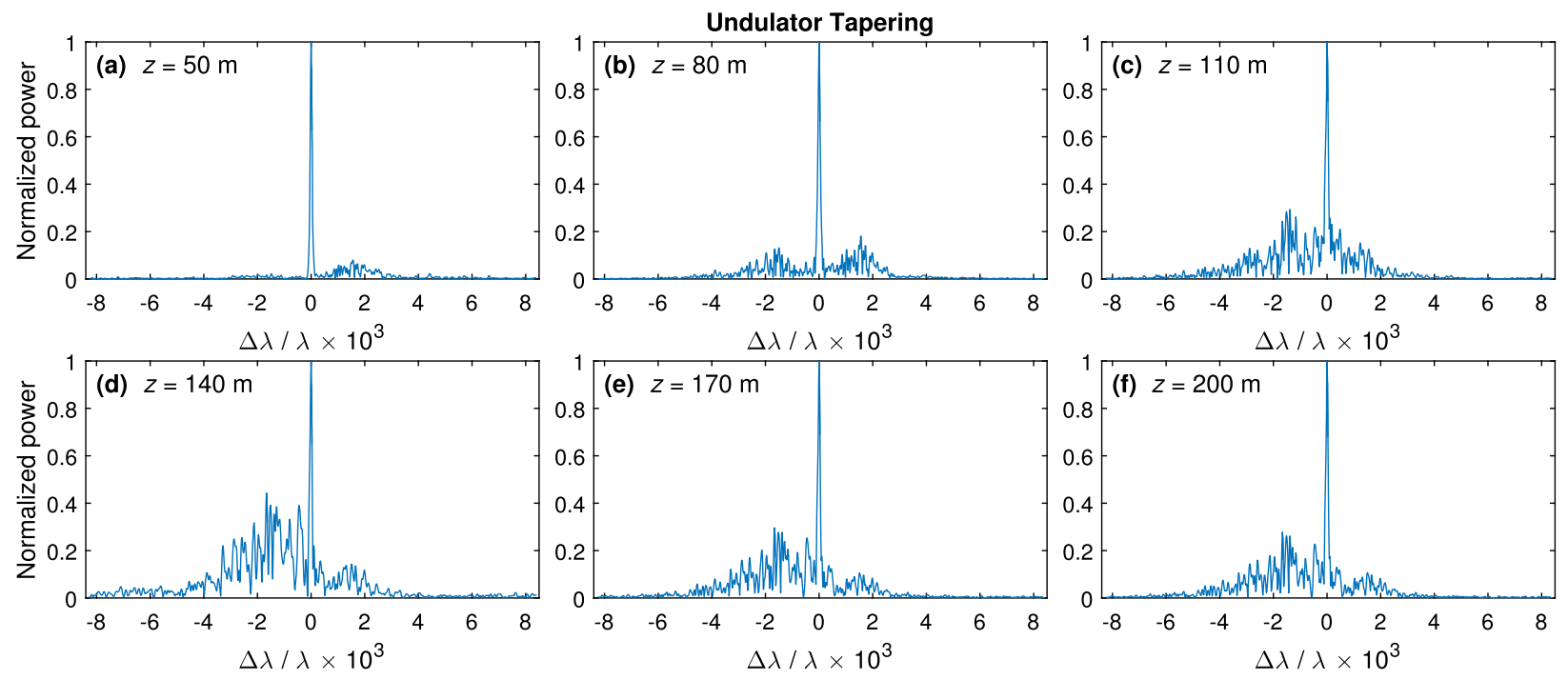

FIG. 11. Result of time-dependent simulation. The evolution of the optical spectrum for the optimal taper is illustrated with the snapshots at six positions along the undulator line.
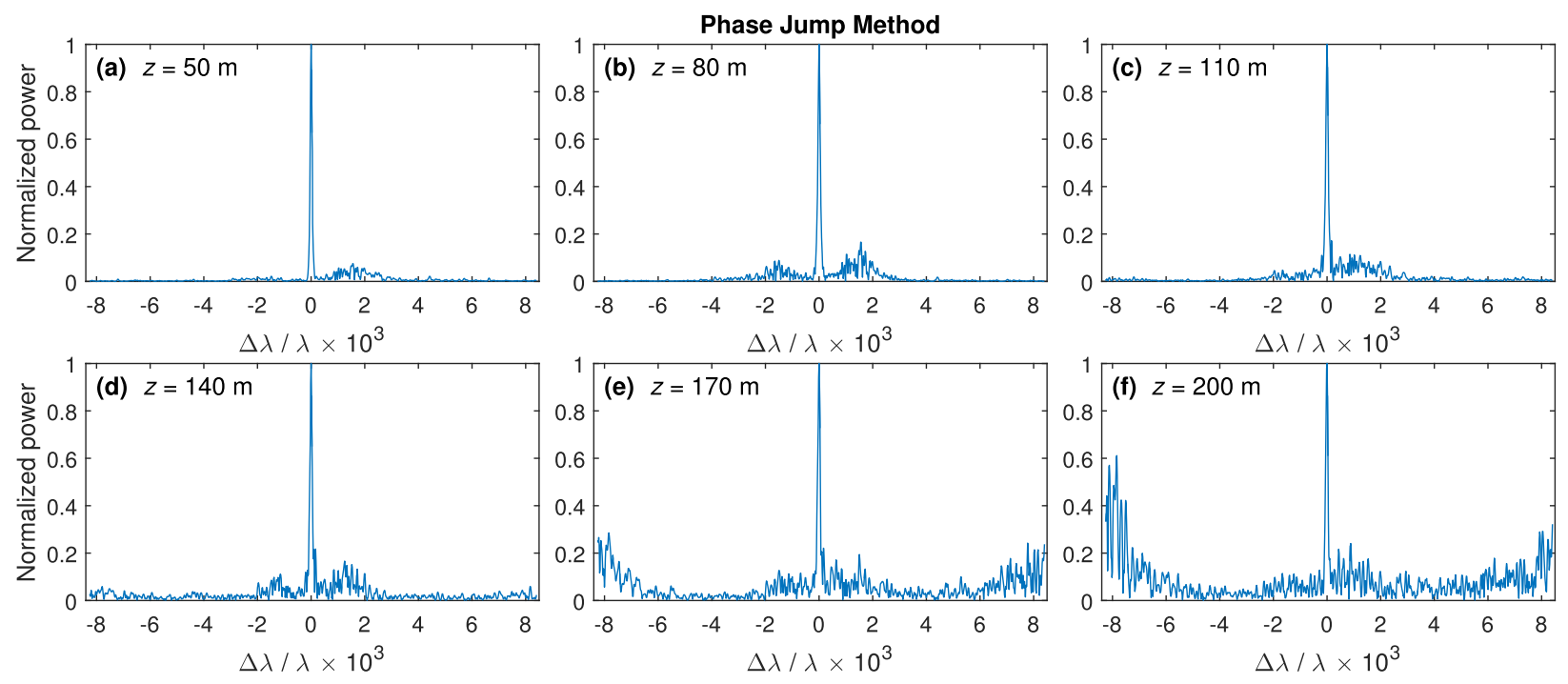

FIG. 12. Result of time-dependent simulation. The evolution of the optical spectrum for the optimal phase jumps is illustrated with the snapshots at six positions along the undulator line.

If we define the FEL efficiency in terms of the absolute spectral power at the central wavelength upon the final saturation, then the phase jump method and undulator tapering are almost equally as efficient in this case.

Between the initial and final saturations, the synchrotron period $L_{\text {sync }}$ has a mean value of $29 \mathrm{~m}$ for the taper, and $30 \mathrm{~m}$ for the phase jumps. The values for the taper and the phase jumps are almost identical. According to Eq. (37), the synchrotron oscillations trigger the growth of sidebands around $\Delta \lambda / \lambda= \pm 1.5 \times 10^{-3}$.

For the taper, there is significant growth of sidebands around these wavelengths after the initial saturation (see Fig. 11). In the vicinity of the final saturation, the sideband power even exceeds $40 \%$ of the central wavelength power [see Fig. 11(d)].

For the phase jumps, there are also sidebands growing around these wavelengths, but the power remains mostly below $20 \%$ of that at the central wavelength (see Fig. 12).

Beyond the final saturation, new sidebands emerge around $\Delta \lambda / \lambda= \pm 8 \times 10^{-3}$ [see Fig. 12(e)-(f)]. However, these new sidebands can be prevented by disengaging all the undulator segments located downstream of the final saturation point.

From these results, it is apparent that the phase jump method is more effective in suppressing the synchrotron sidebands around $\Delta \lambda / \lambda= \pm 1.5 \times 10^{-3}$. Out of the energy extracted from the electron beam, a smaller fraction is 
channeled to these sidebands, and a larger fraction is channeled to the central wavelength.

Hence, if we define the FEL efficiency in terms of the power ratio between the central wavelength and the sideband, then the phase jump method is more efficient than undulator tapering in this case.

\section{E. Phase jumps and the driven oscillator}

It is apparent from Eq. (9) that the synchrotron motion in $\psi$ is mathematically equivalent to the simple pendulum, which is a simple harmonic oscillator at small $\psi$.

The applied phase jumps act as a periodic external driving force, turning the system into a driven oscillator. The period of the driving force is the distance between successive phase shifters, given by $L_{\text {segm }}+L_{\text {drift }}$.

In this driven oscillator, there is a competition between the natural period and the driving period. By disrupting the synchrotron oscillations, the driving force channels power away from the sidebands associated with the natural period, which emerge at optical wavelengths given by Eq. (37). This explains the suppression of these sidebands in Fig. 12.

Beyond the final saturation, the optical field amplitude no longer grows. Furthermore, as the microbunch is out of the bucket, the trajectories in $(\psi, \eta)$ do not form closed orbits, and synchrotron motion no longer has a natural period. The competition is then dominated by the driving force. In analogy to Eq. (37), the periodic driving force can trigger new sidebands at

$$
\frac{\Delta \lambda}{\lambda}= \pm \frac{\lambda_{u}}{L_{\mathrm{segm}}+L_{\mathrm{drift}}}
$$

With the parameter values in Table II, Eq. (38) predicts the new sidebands to occur at $\Delta \lambda / \lambda= \pm 8 \times 10^{-3}$. This agrees with the observation in Fig. 12(e)-(f).

According to Eq. (38), it is, in principle, possible to influence the new sidebands by varying $L_{\text {segm }}$ and $L_{\text {drift }}$ as functions of $z$.

\section{COMBINING PHASE JUMPS AND TAPER}

In our time-dependent simulation, undulator tapering yields a higher optical pulse energy at the final saturation. However, the phase jump method is more effective in suppressing the synchrotron sidebands, thus channeling a larger fraction of the optical energy to the desired central wavelength. This provides a motivation for combining the strengths of the two techniques.

In a recent simulation study by Duris et al. [20], a strong taper is used for the rapid deceleration of electrons, while phase shifters are used for the suppression of synchrotron sidebands.

In that scheme, each phase shift is chosen such that the total slippage in the drift section corresponds to a phase of
$2 n \pi$ with respect to the central wavelength, and $\left(2 n^{\prime}+1\right) \pi$ with respect to the sideband wavelength. Here $n$ and $n^{\prime}$ are positive integers.

In our phase jump method, the total slippage in each drift section corresponds to a phase of $2 n \pi-\psi_{\text {jump }}$ with respect to the central wavelength. The phase shifts used by Duris et al. can therefore be seen as a special case of the phase jump method, where $\psi_{\text {jump }} \equiv 0$.

With $\psi_{\text {jump }} \equiv 0$, the phase shifters do not take part in decelerating the particles. The deceleration relies solely on undulator tapering. As a result, there is no out-of-bucket regime. In general, energy can only be extracted from particles which manage to stay within the rapidly shrinking bucket.

In the simulation study by Duris et al., the value of $n$ ranges from 100 to 330 . This means that the slippage introduced by a phase-shifting chicane can be as large as 330 times the optical wavelength. If the chicane is not isochronous, then the large dispersion can significantly degrade the bunching and the trapping.

In our phase jump method, however, the requirement on the isochronism of the chicane is far less stringent, as the method does not rely on large slippage to suppress the synchrotron sidebands. Instead, the method disrupts the synchrotron oscillations by the microbunch deceleration cycles.

In our time-dependent simulation, $n$ is chosen to be as small as possible, and is always less than 20 . The smallest possible value of $n$ depends on the length of the drift section and the energy of the electron beam.

Beyond this work, a possible subject of further study is the combined use of taper and phase jumps, such that both take part in decelerating the particles, and such that the outof-bucket regime can be exploited.

\section{CONCLUSION}

In this article, we have examined the underlying mechanism of the phase jump method for enhancing the efficiency of an FEL. We have developed a new physics model, and supported it with numerical simulations.

Our model expands beyond previous models by proposing the microbunch deceleration cycle. The model also sets out the selection criteria for the target phase in each phase jump, eliminating the need to deduce the required phase jumps from a preoptimized undulator taper.

In addition, the model opens up the possibility to extract energy from particles outside the ponderomotive bucket, as well as the possibility to suppress the growth of synchrotron sidebands.

In our time-dependent simulation, the phase jump method yields a lower optical pulse energy at the final saturation than undulator tapering. Nonetheless, the fraction of energy channeled to the sidebands is smaller for the phase jump method than for the taper. As a result, the two 
techniques yield almost identical spectral power at the central wavelength near the final saturation.

If we define the FEL efficiency in terms of the spectral power at the central wavelength, then the phase jump method is just as efficient as the taper in this case.

\section{ACKNOWLEDGMENTS}

The simulations presented in this article were performed on resources provided by the Swedish National Infrastructure for Computing (SNIC) at Lund University.

[1] H. H. Lu, Y. Li, and J. Pflueger, The permanent magnet phase shifter for the European X-Ray Free Electron Laser, Nucl. Instrum. Methods Phys. Res., Sect. A 605, 399 (2009).

[2] B. Diviacco, R. Bracco, D. Millo, and M. Musardo, Phase shifters for the FERMI @ Elettra undulators, in Proceedings of the 2nd International Particle Accelerator Conference, San Sebastian, Spain (JACoW, Geneva, Switzerland, 2011), p. 3278.

[3] H. G. Lee, D. E. Kim, W. W. Lee, K. H. Park, S. B. Lee, H. S. Suh, Y. G. Jung, and H. S. Kang, Design and fabrication of prototype phase shifter for PAL XFEL, in Proceedings of the 4th International Particle Accelerator Conference, Shanghai, China (JACoW, Geneva, Switzerland, 2013), p. 3564.

[4] M. Tischer, P. Neumann, A. Schöps, and P. Vagin, Phase shifters for the FLASH2 FEL, in Proceedings of the 5th International Particle Accelerator Conference, Dresden, Germany (JACoW, Geneva, Switzerland, 2014), p. 2010.

[5] B. W. J. McNeil, G. R. M. Robb, M. W. Poole, and N. R. Thompson, Harmonic Lasing in a Free-Electron-Laser Amplifier, Phys. Rev. Lett. 96, 084801 (2006).

[6] E. A. Schneidmiller and M. V. Yurkov, Harmonic lasing in x-ray Free Electron Lasers, Phys. Rev. ST Accel. Beams 15, 080702 (2012).

[7] N. R. Thompson and B. W. J. McNeil, Mode Locking in a Free-Electron Laser Amplifier, Phys. Rev. Lett. 100, 203901 (2008).

[8] A. A. Varfolomeev, T. V. Yarovoi, and P. V. Bousine, Possible enhancement of SASE FEL output field intensity induced by local phase jump, Nucl. Instrum. Methods Phys. Res., Sect. A 407, 296 (1998).

[9] N. M. Kroll, P. L. Morton, and M. N. Rosenbluth, Freeelectron lasers with variable parameter wigglers, IEEE J. Quantum Electron. 17, 1436 (1981).

[10] Y. Jiao, J. Wu, Y. Cai, A. W. Chao, W. M. Fawley, J. Frisch, Z. Huang, H.-D. Nuhn, C. Pellegrini, and S. Reiche, Modeling and multidimensional optimization of a tapered free electron laser, Phys. Rev. ST Accel. Beams 15, 050704 (2012).

[11] A. Mak, F. Curbis, and S. Werin, Model-based optimization of tapered free-electron lasers, Phys. Rev. ST Accel. Beams 18, 040702 (2015).

[12] D. Ratner, Z. Huang, and A. Chao, Enhancing FEL power with phase shifters, in Proceedings of the 29th International Free-Electron Laser Conference, Novosibirsk, Russia (JACoW, Geneva, Switzerland, 2007), p. 69.

[13] J. Wu et al., X-ray spectra and peak power control with iSASE, in Proceedings of the 4th International Particle Accelerator Conference, Shanghai, China (JACoW, Geneva, Switzerland, 2013), p. 2068.

[14] P. Schmüser, M. Dohlus, and J. Rossbach, in Ultraviolet and Soft X-Ray Free-Electron Lasers: Introduction to Physical Principles, Experimental Results, Technological Challenges (Springer, Berlin, Germany, 2008), p. 29.

[15] S. Reiche, GENESIS 1.3: A fully 3D time-dependent FEL simulation code, Nucl. Instrum. Methods Phys. Res., Sect. A 429, 243 (1999).

[16] N. M. Kroll and M. N. Rosenbluth, in Free-Electron Generators of Coherent Radiation, edited by S. F. Jacobs, H. S. Pilloff, M. Sargent, M. O. Scully, and R. Spitzer (Addison-Wesley, Reading, MA, USA, 1980), p. 147.

[17] R. W. Warren, B. E. Newman, and J. C. Goldstein, Raman spectra and the Los Alamos Free-Electron Laser, IEEE J. Quantum Electron. 21, 882 (1985).

[18] D. C. Quimby, J. M. Slater, and J. P. Wilcoxon, Sideband suppression in free-electron lasers with multiple synchrotron periods, IEEE J. Quantum Electron. 21, 979 (1985).

[19] C. Emma, K. Fang, J. Wu, and C. Pellegrini, High efficiency, multiterawatt x-ray free electron lasers, Phys. Rev. Accel. Beams 19, 020705 (2016).

[20] J. Duris, A. Murokh, and P. Musumeci, Tapering enhanced stimulated superradiant amplification, New J. Phys. 17, 063036 (2015). 